TRANSACTIONS OF THE

AMERICAN MATHEMATICAL SOCIETY

Volume 361, Number 7, July 2009, Pages 3375-3399

S 0002-9947(09)04766-7

Article electronically published on March 3, 2009

\title{
THE LOWER CENTRAL AND DERIVED SERIES OF THE BRAID GROUPS OF THE SPHERE
}

\author{
DACIBERG LIMA GONÇALVES AND JOHN GUASCHI
}

\begin{abstract}
In this paper, we determine the lower central and derived series for the braid groups of the sphere. We are motivated in part by the study of Fadell-Neuwirth short exact sequences, but the problem is important in its own right.

The braid groups of the 2-sphere $\mathbb{S}^{2}$ were studied by Fadell, Van Buskirk and Gillette during the 1960s, and are of particular interest due to the fact that they have torsion elements (which were characterised by Murasugi). We first prove that for all $n \in \mathbb{N}$, the lower central series of the $n$-string braid group $B_{n}\left(\mathbb{S}^{2}\right)$ is constant from the commutator subgroup onwards. We obtain a presentation of $\Gamma_{2}\left(B_{n}\left(\mathbb{S}^{2}\right)\right)$, from which we observe that $\Gamma_{2}\left(B_{4}\left(\mathbb{S}^{2}\right)\right)$ is a semi-direct product of the quaternion group $\mathcal{Q}_{8}$ of order 8 by a free group $\mathbb{F}_{2}$ of rank 2. As for the derived series of $B_{n}\left(\mathbb{S}^{2}\right)$, we show that for all $n \geq 5$, it is constant from the derived subgroup onwards. The group $B_{n}\left(\mathbb{S}^{2}\right)$ being finite and soluble for $n \leq 3$, the critical case is $n=4$ for which the derived subgroup is the above semi-direct product $\mathcal{Q}_{8} \rtimes \mathbb{F}_{2}$. By proving a general result concerning the structure of the derived subgroup of a semi-direct product, we are able to determine completely the derived series of $B_{4}\left(\mathbb{S}^{2}\right)$ which from $\left(B_{4}\left(\mathbb{S}^{2}\right)\right)^{(4)}$ onwards coincides with that of the free group of rank 2 , as well as its successive derived series quotients.
\end{abstract}

\section{INTRODUCTION}

1.1. Generalities and definitions. Let $n \in \mathbb{N}$. The braid groups of the plane $\mathbb{E}^{2}$, denoted by $B_{n}$ and known as Artin braid groups, were introduced by E. Artin in 1925 [1, 2, 3] and admit the following well-known presentation: $B_{n}$ is generated by elements $\sigma_{1}, \ldots, \sigma_{n-1}$, subject to the classical Artin relations:

$$
\left\{\begin{array}{l}
\sigma_{i} \sigma_{j}=\sigma_{j} \sigma_{i} \text { if }|i-j| \geq 2 \text { and } 1 \leq i, j \leq n-1, \\
\sigma_{i} \sigma_{i+1} \sigma_{i}=\sigma_{i+1} \sigma_{i} \sigma_{i+1} \text { for all } 1 \leq i \leq n-2 .
\end{array}\right.
$$

A natural generalisation to braid groups of arbitrary topological spaces was made at the beginning of the 1960s by Fox (using the notion of configuration space) [18]. The braid groups of compact, connected surfaces have been widely studied, and (finite) presentations were obtained in [5, 41, 45, 46]. As well as being interesting in their own right, braid groups have played an important rôle in many branches of mathematics; for example in topology, geometry, algebra and dynamical systems,

Received by the editors April 15, 2006.

2000 Mathematics Subject Classification. Primary 20F36, 20F14; Secondary 20F05, 55R80, 20E26.

Key words and phrases. Surface braid group, sphere braid group, lower central series, derived series, configuration space, exact sequence. 
and notably in the study of knots and links [9, of mapping class groups [6, 7], and of configuration spaces [10, 12]. The reader may consult [6, 34, 40] for some general references on the theory of braid groups.

Let $M$ be a connected manifold of dimension 2 (or surface), perhaps with boundary. Further, we shall suppose that $M$ is homeomorphic to a compact 2-manifold with a finite (possibly zero) number of points removed from its interior. We recall two (equivalent) definitions of surface braid groups. The first is due to Fox. Let $F_{n}(M)$ denote the $n^{\text {th }}$ configuration space of $M$, namely the set of all ordered $n$-tuples of distinct points of $M$ :

$$
F_{n}(M)=\left\{\left(x_{1}, \ldots, x_{n}\right) \mid x_{i} \in M \text { and } x_{i} \neq x_{j} \text { if } i \neq j\right\} .
$$

Since $F_{n}(M)$ is a subspace of the $n$-fold Cartesian product of $M$ with itself, the topology on $M$ induces a topology on $F_{n}(M)$. Then we define the $n$-string pure braid group $P_{n}(M)$ of $M$ to be $P_{n}(M)=\pi_{1}\left(F_{n}(M)\right)$. There is a natural action of the symmetric group $S_{n}$ on $F_{n}(M)$ by permutation of coordinates, and the resulting orbit space $F_{n}(M) / S_{n}$ shall be denoted by $D_{n}(M)$. The fundamental group $\pi_{1}\left(D_{n}(M)\right)$ is called the $n$-string (full) braid group of $M$, and shall be denoted by $B_{n}(M)$. Notice that the projection $F_{n}(M) \longrightarrow D_{n}(M)$ is a regular $n$ !-fold covering map.

The second definition of surface braid groups is geometric. Let $\mathcal{P}=\left\{p_{1}, \ldots, p_{n}\right\}$ be a set of $n$ distinct points of $M$. A geometric braid of $M$ with basepoint $\mathcal{P}$ is a collection $\beta=\left(\beta_{1}, \ldots, \beta_{n}\right)$ of $n$ paths $\beta:[0,1] \longrightarrow M$ such that:

(1) for all $i=1, \ldots, n, \beta_{i}(0)=p_{i}$ and $\beta_{i}(1) \in \mathcal{P}$.

(2) for all $i, j=1, \ldots, n$ and $i \neq j$, and for all $t \in[0,1], \beta_{i}(t) \neq \beta_{j}(t)$.

Two geometric braids are said to be equivalent if there exists a homotopy between them through geometric braids. The usual concatenation of paths induces a group operation on the set of equivalence classes of geometric braids. This group is isomorphic to $B_{n}(M)$ and does not depend on the choice of $\mathcal{P}$. The subgroup of pure braids, satisfying additionally $\beta_{i}(1)=p_{i}$ for all $i=1, \ldots, n$, is isomorphic to $P_{n}(M)$. There is a natural surjective homomorphism $\tau: B_{n}(M) \longrightarrow S_{n}$ which to a geometric braid $\beta$ associates the permutation $\tau(\beta)$ defined by $\beta_{i}(1)=p_{\tau(\beta)(i)}$. The kernel is precisely $P_{n}(M)$, and we thus obtain the following short exact sequence:

$$
1 \longrightarrow P_{n}(M) \longrightarrow B_{n}(M) \stackrel{\tau}{\longrightarrow} S_{n} \longrightarrow 1 \text {. }
$$

It is well known that $B_{n}\left(\operatorname{resp} . P_{n}\right)$ is isomorphic to $B_{n}\left(\mathbb{D}^{2}\right)$ (resp. $P_{n}\left(\mathbb{D}^{2}\right)$ ), where $\mathbb{D}^{2}$ is the closed 2-disc.

In this paper, we shall be primarily interested in the braid groups of the 2sphere $\mathbb{S}^{2}$. Along with the braid groups of the real projective plane, they are of particular interest, notably because they have non-trivial centre (which is also the case for the Artin braid groups) and torsion elements (which were characterised by Murasugi [38]). We briefly recall some of their properties [11, 14, 19, 44]. If $\mathbb{D}^{2} \subseteq \mathbb{S}^{2}$ is a topological disc, there is a group homomorphism $\iota: B_{n}\left(\mathbb{D}^{2}\right) \longrightarrow B_{n}\left(\mathbb{S}^{2}\right)$ induced by the inclusion. If $\beta \in B_{n}\left(\mathbb{D}^{2}\right)$, then its image $\iota(\beta)$ shall be denoted simply by $\beta$. It is well known that $B_{n}\left(\mathbb{S}^{2}\right)$ is generated by $\sigma_{1}, \ldots, \sigma_{n-1}$ which are subject to the relations (1.1), plus the following relation, known as the surface relation of $B_{n}\left(\mathbb{S}^{2}\right)$ :

$$
\sigma_{1} \cdots \sigma_{n-2} \sigma_{n-1}^{2} \sigma_{n-2} \cdots \sigma_{1}=1
$$

Hence $B_{n}\left(\mathbb{S}^{2}\right)$ is a quotient of $B_{n}$. The first three sphere braid groups are finite: $B_{1}\left(\mathbb{S}^{2}\right)$ is trivial, $B_{2}\left(\mathbb{S}^{2}\right)$ is cyclic of order 2 , and $B_{3}\left(\mathbb{S}^{2}\right)$ is a $Z S$-metacyclic group 
(a group whose Sylow subgroups, commutator subgroup and commutator quotient group are all cyclic) of order 12 . If $n \geq 4$ then $B_{n}\left(\mathbb{S}^{2}\right)$ is infinite. If $n \geq 3$, the so-called 'full twist' braid $\Delta_{n}=\left(\sigma_{1} \cdots \sigma_{n-1}\right)^{n}$ generates the centre $Z\left(B_{n}\left(\mathbb{S}^{2}\right)\right)$ of $B_{n}\left(\mathbb{S}^{2}\right)$ and is the unique element of $B_{n}\left(\mathbb{S}^{2}\right)$ of order 2 .

Our aim in this paper is to study the lower central and derived series of the braid groups of the sphere. Let us recall some definitions and notation concerning these series. If $G$ is a group, then its lower central series $\left\{\Gamma_{i}(G)\right\}_{i \in \mathbb{N}}$ is defined inductively by $\Gamma_{1}(G)=G$, and $\Gamma_{i+1}(G)=\left[G, \Gamma_{i}(G)\right]$ for all $i \in \mathbb{N}$, and its derived series $\left\{G^{(i)}\right\}_{i \in \mathbb{N} \cup\{0\}}$ is defined inductively by $G^{(0)}=G$, and $G^{(i)}=\left[G^{(i-1)}, G^{(i-1)}\right]$ for all $i \in \mathbb{N}$. One may check easily that $\Gamma_{i}(G) \supseteq \Gamma_{i+1}(G)$ and $G^{(i-1)} \supseteq G^{(i)}$ for all $i \in \mathbb{N}$, and for all $j \in \mathbb{N}, j>i, \Gamma_{j}(G)$ (resp. $\left.G^{(j)}\right)$ is a normal subgroup of $\Gamma_{i}(G)$ (resp. $\left.G^{(i)}\right)$. Notice that $\Gamma_{2}(G)=G^{(1)}$ is the commutator subgroup of $G$. The Abelianisation of the group $G$, denoted by $G^{\mathrm{Ab}}$, is the quotient $G / \Gamma_{2}(G)$; the Abelianisation of an element $g \in G$ is its $\Gamma_{2}(G)$-coset in $G^{\mathrm{Ab}}$. The group $G$ is said to be perfect if $G=G^{(1)}$, or equivalently if $G^{\mathrm{Ab}}=\{1\}$. Following P. Hall, for any group-theoretic property $\mathcal{P}$, a group $G$ is said to be residually $\mathcal{P}$ if for any (nontrivial) element $x \in G$, there exists a group $H$ with the property $\mathcal{P}$ and a surjective homomorphism $\varphi: G \longrightarrow H$ such that $\varphi(x) \neq 1$. It is well known that a group $G$ is residually nilpotent (respectively residually soluble) if and only if $\bigcap_{i \geq 1} \Gamma_{i}(G)=\{1\}$ (respectively $\bigcap_{i \geq 0} G^{(i)}=\{1\}$ ). If $g, h \in G$, then $[g, h]=g h g^{-1} h^{-1}$ will denote their commutator, and we shall use the notation $g \rightleftharpoons h$ to mean that $g$ and $h$ commute.

The lower central series of groups and their successive quotients $\Gamma_{i} / \Gamma_{i+1}$ are isomorphism invariants and have been widely studied using commutator calculus; in particular for free groups of finite rank [33, 37. Falk and Randell, and independently Kohno investigated the lower central series of the pure braid group $P_{n}$ and were able to conclude that $P_{n}$ is residually nilpotent [15, 36]. Falk and Randell also studied the lower central series of generalised pure braid groups [16, 17.

Using the Reidemeister-Schreier rewriting process, Gorin and Lin obtained a presentation of the commutator subgroup of $B_{n}$ for $n \geq 3[32$. For $n \geq 5$, they were able to infer that $\left(B_{n}\right)^{(1)}=\left(B_{n}\right)^{(2)}$, and so conclude that $\left(B_{n}\right)^{(1)}$ is perfect. From this it follows that $\Gamma_{2}\left(B_{n}\right)=\Gamma_{3}\left(B_{n}\right)$; hence $B_{n}$ is not residually nilpotent. If $n=3$, then they showed that $\left(B_{3}\right)^{(1)}$ is a free group of rank 2 , while if $n=4$, they proved that $\left(B_{4}\right)^{(1)}$ is a semi-direct product of two free groups of rank 2 . By considering the action, one may see that $\left(B_{4}\right)^{(1)} \supsetneqq\left(B_{4}\right)^{(2)}$. The work of Gorin and Lin on these series was motivated by the study of almost periodic solutions of algebraic equations with almost periodic coefficients.

The above comments indicate that the study of the lower central and derived series of the braid groups of the sphere is an important problem in its own right, and it enables us to understand better the structure of such groups. But we are also motivated by the interesting question of the existence of a section, or 'splitting problem', for the following two short exact sequences of braid groups (notably for the case $M=\mathbb{S}^{2}$ ) obtained by considering the long exact sequences in homotopy of fibrations of the corresponding configuration spaces:

(1) Let $m, n \in \mathbb{N}$ and $m>n$. Then we have the Fadell-Neuwirth short exact sequence of pure braid groups [13]:

$$
1 \longrightarrow P_{m-n}\left(M \backslash\left\{x_{1}, \ldots, x_{n}\right\}\right) \stackrel{i_{*}}{\longrightarrow} P_{m}(M) \stackrel{p_{*}}{\longrightarrow} P_{n}(M) \longrightarrow 1
$$


where $n \geq 3$ if $M$ is the 2-sphere $\mathbb{S}^{2}$ 11, 14, $n \geq 2$ if $M$ is the real projective plane $\mathbb{R} P^{2}$ [44], and $n \geq 1$ otherwise [13], and where $p_{*}$ is the group homomorphism which geometrically corresponds to forgetting the last $m-n$ strings, and $i_{*}$ is inclusion (we consider $P_{m-n}\left(M \backslash\left\{x_{1}, \ldots, x_{n}\right\}\right.$ ) to be the subgroup of $P_{m}(M)$ of pure braids whose last $n$ strings are vertical). This short exact sequence plays a central rôle in the study of surface braid groups. It was used for example to study mapping class groups [39], Vassiliev invariants for braid groups [31, as well as to obtain presentations for surface pure braid groups [5, 20, 23, 41.

(2) Let $m, n \in \mathbb{N}$. Consider the group homomorphism $\tau: B_{m+n}(M) \longmapsto$ $S_{m+n}$, and let $B_{m, n}(M)=\tau^{-1}\left(S_{m} \times S_{n}\right)$ be the inverse image of the subgroup $S_{m} \times S_{n}$ of $S_{m+n}$. As in the pure braid group case, we obtain a generalisation of the Fadell-Neuwirth short exact sequence [21]:

$$
1 \longrightarrow B_{n}\left(M \backslash\left\{x_{1}, \ldots, x_{m}\right\}\right) \longrightarrow B_{m, n}(M) \stackrel{p_{*}}{\longrightarrow} B_{m}(M) \longrightarrow 1,
$$

where we take $m \geq 3$ if $M=\mathbb{S}^{2}, m \geq 2$ if $M=\mathbb{R} P^{2}$ and $m \geq 1$ otherwise.

Once more, $p_{*}$ corresponds geometrically to forgetting the last $n$ strings.

We remark that if the above conditions on $n$ and $m$ are satisfied, then the existence of a section for $p_{*}$ is equivalent to that of a geometric section for the corresponding configuration spaces (cf. 22, 23]). If $M$ is the plane, then the fact that (1.3) splits for all $n \in \mathbb{N}$ implies that $P_{n}$ may be expressed as a repeated semidirect product of free groups [2], which leads to a solution of the word problem for $P_{n}$ and $B_{n}$. The splitting problem has been studied for other surfaces besides the plane. Fadell and Neuwirth gave various sufficient conditions for the existence of a geometric section for $p$ in the general case [13. For the sphere, it was known that there exists a section on the geometric level [14. If $M$ is the 2-torus, then Birman exhibited an explicit algebraic section for (1.3) for $m=n+1$ and $n \geq 2$ [5]. However, for compact orientable surfaces without boundary of genus $g \geq 2$, she posed the question of whether the short exact sequence (1.3) splits. In [20, we provided a complete answer to this question:

Theorem 1.1 (20]). If $M$ is a compact orientable surface without boundary of genus $g \geq 2$, the short exact sequence (1.3) splits if and only if $n=1$.

In the case of $\mathbb{R} P^{2}$, Van Buskirk showed that the exact sequence (1.3) splits if $m=3$ and $n=2$ [44. We recently showed that this condition is also necessary, and thus answered a question posed by Van Buskirk in that paper:

Theorem 1.2 (25]). Let $M$ be the real projective plane $\mathbb{R} P^{2}$. Then the short exact sequence (1.3) splits if and only if $m=3$ and $n=2$.

In [23], we studied the short exact sequence (1.4) in the case $M=\mathbb{S}^{2}$ of the sphere, and showed that if $m=3$, then the short exact sequence (1.4) splits if and only if $n \equiv 0,2 \bmod 3$. Further, if $m \geq 4$ and the short exact sequence (1.4) splits, then there exist $\varepsilon_{1}, \varepsilon_{2} \in\{0,1\}$ such that $n \equiv \varepsilon_{1}(m-1)(m-2)-\varepsilon_{2} m(m-2)$ $(\bmod m(m-1)(m-2))$. An open question is whether this condition is also sufficient.

The study in this paper of the lower central and derived series of the braid groups of the sphere was motivated in part by the question of the existence of a section for the short exact sequences (1.3) and (1.4). To obtain a positive answer, it suffices of course to exhibit an explicit section (although this may be easier said than done!). However, and in spite of the fact that we possess presentations of surface braid 
groups, in general it is very difficult to prove directly that such an extension does not split. One of the main methods that we used to prove the non-splitting of (1.3) for $n \geq 2$ and of (1.4) for $m \geq 4$ was based on the following observation: let $1 \longrightarrow K \longrightarrow G \longrightarrow Q \longrightarrow 1$ be a split extension of groups, where $K$ is a normal subgroup of $G$, and let $H$ be a normal subgroup of $G$ contained in $K$. Then the extension $1 \longrightarrow K / H \longrightarrow G / H \longrightarrow Q \longrightarrow 1$ also splits. The condition on $H$ is satisfied for example if $H$ is an element of either the lower central series or the derived series of $K$. In [20], considering the extension (1.3) with $n \geq 3$, we showed that it was sufficient to take $H=\Gamma_{2}(K)$ to prove the non-splitting of the quotiented extension, and hence that of the full extension. In this case, the kernel $K / \Gamma_{2}(K)$ is Abelian, which simplifies somewhat the calculations in $G / H$. This was also the case in 23] for the extension (1.4) with $m \geq 4$. However, for the extension (1.3) with $n=2$, it was necessary to go a stage further in the lower central series and take $H=\Gamma_{3}(K)$. From the point of view of the splitting problem, it is thus helpful to know the lower central and derived series of the braid groups occurring in these group extensions. But as we indicated earlier, these series are of course interesting in their own right and help us to understand better the structure of surface braid groups.

1.2. Statement of the main results. This paper is organised as follows. In Section 2.1, we prove some general results regarding the splitting of the short exact sequence $1 \longrightarrow \Gamma_{2}\left(B_{n}\left(\mathbb{S}^{2}\right)\right) \longrightarrow B_{n}\left(\mathbb{S}^{2}\right) \longrightarrow\left(B_{n}\left(\mathbb{S}^{2}\right)\right)$ Ab $\longrightarrow 1$, as well as homological conditions for the stabilisation of the lower central series of a group (Lemma 2.4).

In Section 2.2, we prove Theorem 1.3, which deals with the lower central series of $B_{n}\left(\mathbb{S}^{2}\right)$ :

Theorem 1.3. For all $n \geq 2$, the lower central series of $B_{n}\left(\mathbb{S}^{2}\right)$ is constant from the commutator subgroup onwards: $\Gamma_{m}\left(B_{n}\left(\mathbb{S}^{2}\right)\right)=\Gamma_{2}\left(B_{n}\left(\mathbb{S}^{2}\right)\right)$ for all $m \geq 2$. The subgroup $\Gamma_{2}\left(B_{n}\left(\mathbb{S}^{2}\right)\right)$ is as follows:

(1) If $n=1,2$, then $\Gamma_{2}\left(B_{n}\left(\mathbb{S}^{2}\right)\right)=\{1\}$.

(2) If $n=3$, then $\Gamma_{2}\left(B_{n}\left(\mathbb{S}^{2}\right)\right) \cong \mathbb{Z}_{3}$. Thus $B_{3}\left(\mathbb{S}^{2}\right) \cong \mathbb{Z}_{3} \rtimes \mathbb{Z}_{4}$, the action being the non-trivial one.

(3) If $n=4$, then $\Gamma_{2}\left(B_{4}\left(\mathbb{S}^{2}\right)\right)$ admits a presentation of the following form:

generators: $g_{1}, g_{2}, g_{3}$, where in terms of the usual generators of $B_{4}\left(\mathbb{S}^{2}\right), g_{1}=$ relations:

$$
\sigma_{1}^{2} \sigma_{2} \sigma_{1}^{-3}, g_{2}=\sigma_{1}^{3} \sigma_{2} \sigma_{1}^{-4} \text { and } g_{3}=\sigma_{3} \sigma_{1}^{-1} \text {. }
$$

$$
\begin{gathered}
g_{3}^{4}=1 \\
{\left[g_{3}^{2}, g_{i}\right]=1 \text { for } i=1,2,} \\
{\left[g_{3}, g_{2} g_{1}\right]=1} \\
g_{1}^{-1} g_{3}^{-1} g_{1}=g_{2} g_{3} g_{2}^{-1} \\
g_{1}^{-1} g_{3}^{-1} g_{1}=g_{1} g_{3} g_{1}^{-1} g_{3}
\end{gathered}
$$

Furthermore,

$$
\Gamma_{2}\left(B_{4}\left(\mathbb{S}^{2}\right)\right) \cong \mathcal{Q}_{8} \rtimes \mathbb{F}_{2}(a, b),
$$

where $\mathcal{Q}_{8}=\left\langle x, y \mid x^{2}=y^{2}, x y x^{-1}=y^{-1}\right\rangle$ is the quaternion group of order 8 , and $\mathbb{F}_{2}(a, b)$ is the free group of rank 2 on two generators a 
and $b$. The following elements of $B_{4}\left(\mathbb{S}^{2}\right)$ realise these subgroups: $x=g_{3}$, $y=g_{1} g_{3} g_{1}^{-1}, a=g_{1}$ and $b=g_{2}$. The action is given by:

$$
\begin{array}{ll}
\varphi(a)(x)=y, & \varphi(a)(y)=x y, \\
\varphi(b)(x)=y x, & \varphi(b)(y)=x .
\end{array}
$$

(4) If $n \geq 4$, a presentation for $\Gamma_{2}\left(B_{n}\left(\mathbb{S}^{2}\right)\right)$ is as given in Proposition 4.1 (see Section 4.1).

The lower central series of $B_{n}\left(\mathbb{S}^{2}\right)$ is thus completely determined. In particular, for all $n \geq 2$, the lower central series of $B_{n}\left(\mathbb{S}^{2}\right)$ is constant from the commutator subgroup onwards, and $B_{n}\left(\mathbb{S}^{2}\right)$ is residually nilpotent if and only if $n \leq 2$. The case $n=4$ is particularly interesting: $\Gamma_{2}\left(B_{4}\left(\mathbb{S}^{2}\right)\right)$ is a semi-direct product of the quaternion group $\mathcal{Q}_{8}$ of order 8 by the free group of rank 2 . This may be compared with Gorin and Lin's result for $\Gamma_{2}\left(B_{4}\right)$ 32. Thus $B_{4}\left(\mathbb{S}^{2}\right)$ contains an isomorphic copy of $\mathcal{Q}_{8}$. We learnt that this inclusion had previously been studied by Thompson [43. We then showed that for all $n \geq 3, B_{n}\left(\mathbb{S}^{2}\right)$ contains an isomorphic copy of $\mathcal{Q}_{8}$ of order 8 if and only if $n$ is even [24. This leads naturally to the interesting problems of the classification of the finite and virtually cyclic subgroups of $B_{n}\left(\mathbb{S}^{2}\right)$ and $B_{n}\left(\mathbb{R} P^{2}\right)$. We have recently classified the finite subgroups of $B_{n}\left(\mathbb{S}^{2}\right)$ [25] and the finite and virtually cyclic subgroups of $P_{n}\left(\mathbb{R} P^{2}\right)[26$. We are currently pursuing the study of the virtually cyclic subgroups of $B_{n}\left(\mathbb{S}^{2}\right)[29$, as well as the finite and virtually cyclic subgroups of $B_{n}\left(\mathbb{R} P^{2}\right)$ [30].

In Section 3, we study the derived series of $B_{n}\left(\mathbb{S}^{2}\right)$. As in the case of $B_{n}$ [32], $\left(B_{n}\left(\mathbb{S}^{2}\right)\right)^{(1)}$ is perfect if $n \geq 5$; in other words, the derived series of $B_{n}\left(\mathbb{S}^{2}\right)$ is constant from $\left(B_{n}\left(\mathbb{S}^{2}\right)\right)^{(1)}$ onwards. The cases $n=1,2,3$ are straightforward, and the groups $B_{n}\left(\mathbb{S}^{2}\right)$ are finite and soluble. In the case $n=4$, we make use of the semi-direct product decomposition of $\left(B_{4}\left(\mathbb{S}^{2}\right)\right)^{(1)}$ obtained in Theorem 1.3. Proposition 3.3 describes the structure of the commutator subgroup of a general semi-direct product, and it seems to be a useful and interesting result. This enables us to show that from $\left(B_{4}\left(\mathbb{S}^{2}\right)\right)^{(4)}$ onwards, the derived series of $B_{4}\left(\mathbb{S}^{2}\right)$ coincides with that of the free group of rank 2 . We also determine some of the derived series quotients of $B_{4}\left(\mathbb{S}^{2}\right)$ :

Theorem 1.4. The derived series of $B_{n}\left(\mathbb{S}^{2}\right)$ is as follows:

(1) If $n=1,2$, then $\left(B_{n}\left(\mathbb{S}^{2}\right)\right)^{(1)}=\{1\}$.

(2) If $n=3$, then $\left(B_{3}\left(\mathbb{S}^{2}\right)\right)^{(1)} \cong \mathbb{Z}_{3}$ and $\left(B_{3}\left(\mathbb{S}^{2}\right)\right)^{(2)}=\{1\}$.

(3) Suppose that $n=4$. Then:

(a) $\left(B_{4}\left(\mathbb{S}^{2}\right)\right)^{(1)}=\Gamma_{2}\left(B_{4}\left(\mathbb{S}^{2}\right)\right)$ is given by part (3) of Theorem 1.3; it is isomorphic to the semi-direct product $\mathcal{Q}_{8} \rtimes \mathbb{F}_{2}$. Further, the quotient $B_{4}\left(\mathbb{S}^{2}\right) /\left(B_{4}\left(\mathbb{S}^{2}\right)\right)^{(1)}$ is isomorphic to $\mathbb{Z}_{6}$.

(b) $\left(B_{4}\left(\mathbb{S}^{2}\right)\right)^{(2)}$ is isomorphic to the semi-direct product $\mathcal{Q}_{8} \rtimes \mathbb{F}_{2}^{(1)}$, where $\left(\mathbb{F}_{2}\right)^{(1)}$ is the commutator subgroup of the free group $\mathbb{F}_{2}=\mathbb{F}_{2}(a, b)$ of rank 2 on two generators $a, b$. The action of $\left(\mathbb{F}_{2}\right)^{(1)}$ on $\mathcal{Q}_{8}$ is the restriction of the action of $\mathbb{F}_{2}(a, b)$ given in part (3) of Theorem 1.3 . Further,

$\left(B_{4}\left(\mathbb{S}^{2}\right)\right)^{(1)} /\left(B_{4}\left(\mathbb{S}^{2}\right)\right)^{(2)} \cong \mathbb{Z}^{2}$ and $B_{4}\left(\mathbb{S}^{2}\right) /\left(B_{4}\left(\mathbb{S}^{2}\right)\right)^{(2)} \cong \mathbb{Z}^{2} \rtimes \mathbb{Z}_{6}$,

where the action of the generator $\bar{\sigma}$ of $\mathbb{Z}_{6}$ on $\mathbb{Z}^{2}$ is given by left multiplication by the matrix $\left(\begin{array}{cc}0 & 1 \\ -1 & 1\end{array}\right)$. 
(c) $\left(B_{4}\left(\mathbb{S}^{2}\right)\right)^{(3)}$ is a subgroup of $P_{4}\left(\mathbb{S}^{2}\right)$ isomorphic to the direct product $\mathbb{Z}_{2} \times\left(\mathbb{F}_{2}\right)^{(2)}$. Further,

$$
\left(B_{4}\left(\mathbb{S}^{2}\right)\right)^{(2)} /\left(B_{4}\left(\mathbb{S}^{2}\right)\right)^{(3)} \cong\left(\mathbb{Z}_{2} \times \mathbb{Z}_{2}\right) \times\left(\mathbb{F}_{2}\right)^{(1)} /\left(\mathbb{F}_{2}\right)^{(2)} .
$$

(d) $\left(B_{4}\left(\mathbb{S}^{2}\right)\right)^{(m)} \cong\left(\mathbb{F}_{2}\right)^{(m-1)}$ for all $m \geq 4$. Further,

$$
\left(B_{4}\left(\mathbb{S}^{2}\right)\right)^{(3)} /\left(B_{4}\left(\mathbb{S}^{2}\right)\right)^{(4)} \cong \mathbb{Z}_{2} \times\left(\mathbb{F}_{2}\right)^{(2)} /\left(\mathbb{F}_{2}\right)^{(3)},
$$

and for $m \geq 4$,

$$
\left(B_{4}\left(\mathbb{S}^{2}\right)\right)^{(m)} /\left(B_{4}\left(\mathbb{S}^{2}\right)\right)^{(m+1)} \cong\left(\mathbb{F}_{2}\right)^{(m-1)} /\left(\mathbb{F}_{2}\right)^{(m)} .
$$

(4) If $n \geq 5$, then $\left(B_{n}\left(\mathbb{S}^{2}\right)\right)^{(2)}=\left(B_{n}\left(\mathbb{S}^{2}\right)\right)^{(1)}$, so $\left(B_{n}\left(\mathbb{S}^{2}\right)\right)^{(1)}$ is perfect. $A$ presentation of $\left(B_{n}\left(\mathbb{S}^{2}\right)\right)^{(1)}$ is given in Proposition 4.1 .

So the derived series of $B_{n}\left(\mathbb{S}^{2}\right)$ is thus completely determined (up to knowing the derived series of the free group $\mathbb{F}_{2}$ of rank 2; see Remark 3.1). In particular, $B_{n}\left(\mathbb{S}^{2}\right)$ is residually soluble if and only if $n \leq 4$ (Corollary 3.2). Thus $B_{4}\left(\mathbb{S}^{2}\right)$ is residually soluble, but not residually nilpotent.

Finally in Section 4, for $n \geq 4$, we give presentations of the commutator subgroups $\Gamma_{2}\left(B_{n}\left(\mathbb{S}^{2}\right)\right)$ of the sphere braid groups, and in the case $n=4$, in Proposition 4.3 we derive the presentation of $\Gamma_{2}\left(B_{4}\left(\mathbb{S}^{2}\right)\right)$ given in Theorem 1.3(3).

In a companion paper [27], we study the lower central and derived series of the class of braid groups of the finitely-punctured sphere. This class includes the Artin braid groups, the braid groups of the annulus, which are Artin groups of type $B$ (for $n=2$ ), and affine Artin groups of type $\widetilde{C}$ (for $n=3$ ). We remark that since work on these two papers started, one of the authors in collaboration with P. Bellingeri and S. Gervais has undertaken the study of the lower central series of braid groups of orientable surfaces, with and without boundary, of genus $g \geq 1$ [4, and that some of the techniques appearing in this paper were subsequently used there. It is worth stating the following result of [4] which contrasts somewhat with that obtained here for the sphere.

Theorem 1.5 (4]). Let $M$ be a compact, connected orientable surface without boundary, of genus $g \geq 1$, and let $m \geq 3$. Then:

(1) $\Gamma_{1}\left(B_{m}(M)\right) / \Gamma_{2}\left(B_{m}(M)\right) \cong \mathbb{Z}^{2 g} \oplus \mathbb{Z}_{2}$.

(2) $\Gamma_{2}\left(B_{m}(M)\right) / \Gamma_{3}\left(B_{m}(M)\right) \cong \mathbb{Z}_{m-1+g}$.

(3) $\Gamma_{3}\left(B_{m}(M)\right)=\Gamma_{4}\left(B_{m}(M)\right)$. Moreover, $\Gamma_{3}\left(B_{m}(M)\right)$ is perfect for $m \geq 5$.

This implies that braid groups of compact, connected orientable surfaces without boundary may be distinguished by their lower central series (indeed by the first two lower central quotients). Notice that the lower central series stabilises one stage further, i.e. from $\Gamma_{3}$ onwards, as opposed to $\Gamma_{2}$ in the case of the sphere.

\section{The LOWER CENTRAL SERIES OF $B_{n}\left(\mathbb{S}^{2}\right)$}

The main aim of this section is to prove Theorem 1.3, which describes the lower central series of $B_{n}\left(\mathbb{S}^{2}\right)$. This will be carried out in Section 2.2. Before doing so, in Section 2.1, we state and prove some general results concerning the splitting of the short exact sequence (2.1) (Proposition 2.1), as well as homological conditions for the stabilisation of the lower central series of a group (Lemma 2.4). 
2.1. Generalities. Let $n \in \mathbb{N}$. Let $B_{n}\left(\mathbb{S}^{2}\right)$ denote the braid group of $\mathbb{S}^{2}$ on $n$ strings, let $\left(B_{n}\left(\mathbb{S}^{2}\right)\right){ }^{\mathrm{Ab}}=B_{n}\left(\mathbb{S}^{2}\right) / \Gamma_{2}\left(B_{n}\left(\mathbb{S}^{2}\right)\right)$ denote Abelianisation of $B_{n}\left(\mathbb{S}^{2}\right)$, and let $\alpha: B_{n}\left(\mathbb{S}^{2}\right) \longrightarrow\left(B_{n}\left(\mathbb{S}^{2}\right)\right)$ Ab be the canonical projection. Then we have the following short exact sequence:

$$
1 \longrightarrow \Gamma_{2}\left(B_{n}\left(\mathbb{S}^{2}\right)\right) \longrightarrow B_{n}\left(\mathbb{S}^{2}\right) \stackrel{\alpha}{\longrightarrow}\left(B_{n}\left(\mathbb{S}^{2}\right)\right){ }^{\mathrm{Ab}} \longrightarrow 1 .
$$

We first prove the following result which deals with the splitting of this short exact sequence.

Proposition 2.1. Let $n \in \mathbb{N}$. Then:

(1) $\left(B_{n}\left(\mathbb{S}^{2}\right)\right)^{A b}=B_{n}\left(\mathbb{S}^{2}\right) / \Gamma_{2}\left(B_{n}\left(\mathbb{S}^{2}\right)\right) \cong \mathbb{Z}_{2(n-1)}$.

(2) The short exact sequence (2.1) splits if and only if $n$ is odd, where the action on $\Gamma_{2}\left(B_{n}\left(\mathbb{S}^{2}\right)\right)$ by a generator of $\mathbb{Z}_{2(n-1)}$ is given by conjugation by $\sigma_{1} \ldots \sigma_{n-2} \sigma_{n-1}^{2}$.

(3) If $n$ is even, then $B_{n}\left(\mathbb{S}^{2}\right)$ is not isomorphic to the semi-direct product of a subgroup $K$ by $\mathbb{Z}_{2(n-1)}$.

Proof. (1) This follows easily from the presentation (1.2) of the group $B_{n}\left(\mathbb{S}^{2}\right)$. The generators $\sigma_{i}$ of $B_{n}\left(\mathbb{S}^{2}\right)$ are all identified by $\alpha$ to a single generator $\widetilde{\sigma}=\alpha\left(\sigma_{i}\right)$ of $\mathbb{Z}_{2(n-1)}$.

(2) In order to construct a section, we consider the elements of $B_{n}\left(\mathbb{S}^{2}\right)$ of order 2(n-1). According to Murasugi's classification of the torsion elements of $B_{n}\left(\mathbb{S}^{2}\right)[38$, these elements are precisely the conjugates of the elements of the form $\left(\sigma_{1} \cdots \sigma_{n-2} \sigma_{n-1}^{2}\right)^{r}$, where $r$ and $2(n-1)$ are coprime. Such an element projects to $\widetilde{\sigma}^{r n}$ whose order is $2(n-1) / \operatorname{gcd}(r n, 2(n-1))$. Since

$$
\operatorname{gcd}(r n, 2(n-1))=\operatorname{gcd}(n, 2(n-1))=\operatorname{gcd}(n, 2),
$$

the result follows from equation (2.1) and part (1).

(3) Let $n \in \mathbb{N}$ be even. We first prove the following lemma:

Lemma 2.2. Let $G$ be a group whose Abelianisation $G^{A b}$ is Hopfian, i.e. $G^{A b}$ is not isomorphic to any of its proper quotients. Suppose that there exists a group $H$ isomorphic to $G^{A b}$, a normal subgroup $K$ of $G$, and a split short exact sequence $1 \longrightarrow K \longrightarrow G \longrightarrow H \longrightarrow 1$. Then $G \cong \Gamma_{2}(G) \rtimes G^{A b}$.

Proof of Lemma 2.2, Let $\alpha: G \longrightarrow G^{\mathrm{Ab}}$ denote the Abelianisation homomorphism, let $\xi: G \longrightarrow H$ denote the homomorphism in the given short exact sequence, and let $s: H \longrightarrow G$ be a section for $\xi$. Since $H \cong G / K$ is Abelian, it follows from standard properties of the commutator subgroup that $\Gamma_{2}(G) \subseteq K$. Hence we have the following commutative diagram:

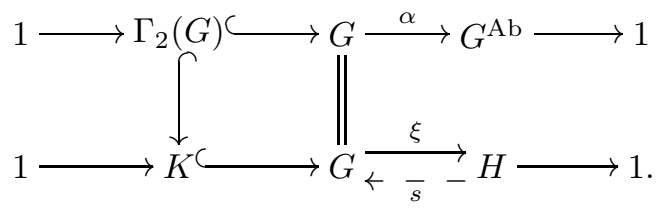

This extends to a commutative diagram of short exact sequences by taking $\rho: G^{\mathrm{Ab}}$ $\longrightarrow H$ defined by $\rho(y)=\xi(x)$ for all $y \in G^{\mathrm{Ab}}$, where $x \in G$ is any element satisfying $\alpha(x)=y$. This homomorphism is well defined, and is surjective since $\xi$ and $\alpha$ are surjective. But $G^{\mathrm{Ab}} \cong H$ is Hopfian by hypothesis, which implies that $\rho$ is an 
isomorphism. Hence $\alpha=\rho^{-1} \circ \xi$, and $s \circ \rho$ is a section for $\alpha$, which proves the lemma.

By taking $G=B_{n}\left(\mathbb{S}^{2}\right)$ and $K=\mathbb{Z}_{2(n-1)}$ in the statement of Lemma 2.2. if $B_{n}\left(\mathbb{S}^{2}\right)$ were a semi-direct product of $K$ with $H$, then this would contradict part (2). This completes the proof of Proposition 2.1.

Remark 2.3. If $n$ is even, let us consider the natural projection $p: \mathbb{Z}_{2(n-1)} \longrightarrow \mathbb{Z}_{n-1}$. Then we have a short exact sequence:

$$
1 \longrightarrow \Gamma_{2}^{*}\left(B_{n}\left(\mathbb{S}^{2}\right)\right) \longrightarrow B_{n}\left(\mathbb{S}^{2}\right) \stackrel{\alpha^{*}}{\longrightarrow} \mathbb{Z}_{(n-1)} \longrightarrow 1,
$$

where $\alpha^{*}=p \circ \alpha$, and $\Gamma_{2}^{*}\left(B_{n}\left(\mathbb{S}^{2}\right)\right)$ is the kernel of $\alpha^{*}$. It is not difficult to see that this short exact sequence splits: a section is given by sending the generator of $\mathbb{Z}_{(n-1)}$ to $\left(\sigma_{1} \ldots \sigma_{n-2} \sigma_{n-1}^{2}\right)^{2^{r}}$, where $2^{r}$ is the greatest power of 2 dividing $n$.

Let $G$ be a group which acts on a group $H$. Following [35, page 67], we may define the commutator subgroup with respect to this action by

$$
\Gamma_{G}(H)=\left\langle(g \star h) k h^{-1} k^{-1} \mid g \in G, h, k \in H\right\rangle,
$$

where $g \star h$ denotes the action of $g$ on $h$. We say that the action is perfect if $\Gamma_{G}(H)=H$. Note that if $H$ is a normal subgroup of $G$, then $H \supseteq \Gamma_{G}(H)=$ $[G, H] \supseteq[H, H]$ for the action of conjugation of $G$ on $H$. In particular, if $G=H$, then $\Gamma_{G}(H)=\Gamma_{2}(G)$ for the action of conjugation of $G$ on itself. If this action is perfect, then the group $G$ is perfect.

Lemma 2.4. Let $G$ be a group, and let $G^{A b}$ be its Abelianisation. Consider the homomorphism $\delta: H_{2}(G, \mathbb{Z}) \longrightarrow H_{2}\left(G^{A b}, \mathbb{Z}\right)$ induced by Abelianisation. Then

$$
\Gamma_{2}(G) / \Gamma_{3}(G) \cong \operatorname{Coker}(\delta) \cong H_{0}\left(G^{A b}, H_{1}\left(\Gamma_{2}(G), \mathbb{Z}\right)\right) .
$$

In particular:

(1) $\Gamma_{2}(G)=\Gamma_{3}(G)$ if and only if $\delta$ is surjective.

(2) If $\mathrm{H}_{2}\left(G^{A b}, \mathbb{Z}\right)$ is trivial, then $\Gamma_{n}(G)=\Gamma_{2}(G)$ for all $n \geq 2$.

(3) If either the action (by conjugation) of $G$ on $\Gamma_{2}(G)$ or the action (by conjugation) of $G^{A b}$ on $H_{1}\left(\Gamma_{2}(G), \mathbb{Z}\right)$ is perfect, then $\Gamma_{n}(G)=\Gamma_{2}(G)$ for all $n \geq 2$.

Proof. Recall that if $1 \longrightarrow K \longrightarrow G \longrightarrow Q \longrightarrow 1$ is an extension of groups, then we have a 6-term exact sequence due to Stallings [8, 42]:

$$
H_{2}(G) \longrightarrow H_{2}(Q) \longrightarrow K /[G, K] \longrightarrow H_{1}(G) \longrightarrow H_{1}(Q) \longrightarrow 1
$$

Applying this to the short exact sequence

$$
1 \longrightarrow \Gamma_{2}(G) \longrightarrow G \longrightarrow G^{\mathrm{Ab}} \longrightarrow 1,
$$

we obtain

$$
H_{2}(G, \mathbb{Z}) \stackrel{\delta}{\longrightarrow} H_{2}\left(G^{\mathrm{Ab}}, \mathbb{Z}\right) \longrightarrow \Gamma_{2}(G) / \Gamma_{3}(G) \longrightarrow H_{1}(G, \mathbb{Z}) \longrightarrow G^{\mathrm{Ab}} \longrightarrow 1 .
$$

But $H_{1}(G, \mathbb{Z}) \longrightarrow G^{\mathrm{Ab}}$ is an isomorphism, so this becomes

$$
H_{2}(G, \mathbb{Z}) \stackrel{\delta}{\longrightarrow} H_{2}\left(G^{\mathrm{Ab}}, \mathbb{Z}\right) \longrightarrow \Gamma_{2}(G) / \Gamma_{3}(G) \longrightarrow 1 .
$$

Hence $\Gamma_{2}(G) / \Gamma_{3}(G) \cong$ Coker $(\delta)$, which yields the first isomorphism. To obtain the second, we consider the Lyndon-Hochschild-Serre spectral sequence [8] applied to the short exact sequence (2.4), for which the relevant terms are $E_{(2,0)}^{2}=H_{2}\left(G^{\mathrm{Ab}}, \mathbb{Z}\right)$ 
and $E_{(0,1)}^{2}=H_{0}\left(G^{\mathrm{Ab}}, H_{1}\left(\Gamma_{2}(G), \mathbb{Z}\right)\right)$. Since $H_{1}(G)=H_{1}\left(G^{\mathrm{Ab}}\right)$, the differential $d_{2}: E_{(2,0)}^{2} \longrightarrow E_{(0,1)}^{2}$ is surjective, with kernel $E_{(2,0)}^{\infty}$. From the general definition of the filtration of $H_{2}(G)$ given by the spectral sequence, we have a surjection $H_{2}(G) \longrightarrow E_{(2,0)}^{\infty}$, and hence the following exact sequence:

$$
H_{2}(G) \longrightarrow E_{(2,0)}^{\infty} \longrightarrow E_{(2,0)}^{2} \longrightarrow E_{(0,1)}^{2} \longrightarrow 1
$$

Hence $\operatorname{Im}(\delta)=E_{(2,0)}^{\infty}$, and

$$
\operatorname{Coker}(\delta)=E_{(2,0)}^{2} / \operatorname{Im}(\delta) \cong E_{(0,1)}^{2}=H_{0}\left(G^{\mathrm{Ab}}, H_{1}\left(\Gamma_{2}(G), \mathbb{Z}\right)\right)
$$

as required. From the first isomorphism, one may check that part (1) is satisfied. Part (2) then follows easily.

To prove part (3), if the action by conjugation of $G$ on $\Gamma_{2}(G)$ is perfect, then $\Gamma_{G}\left(\Gamma_{2}(G)\right)=\left[G, \Gamma_{2}(G)\right]=\Gamma_{3}(G)=\Gamma_{2}(G)$, and the result is clear. Now let us consider the action of $G$ on $H_{1}\left(\Gamma_{2}(G)\right)=\left(\Gamma_{2}(G)\right)^{\text {Ab }}$ given by conjugation, defined by $g \cdot \widetilde{h}=\widetilde{g h g^{-1}}$, where $g, h \in G$ and $\sim$ denotes Abelianisation in $\Gamma_{2}(G)$. If $g \in$ $\Gamma_{2}(G)$, then the induced action on $\left(\Gamma_{2}(G)\right)^{\mathrm{Ab}}$ is trivial, so the original action factors through $G^{\mathrm{Ab}}$, and we obtain an action of $G^{\mathrm{Ab}}$ on $\left(\Gamma_{2}(G)\right)^{\mathrm{Ab}}$ given by $\widetilde{g} \cdot \widetilde{h}=\widetilde{g h g^{-1}}$ $(\widetilde{g}$ denotes the Abelianisation of $g$ in $G)$. Suppose that this action is perfect, so that $\Gamma_{G} \mathrm{Ab}\left(\left(\Gamma_{2}(G)\right)^{\mathrm{Ab}}\right)=\left(\Gamma_{2}(G)\right)^{\mathrm{Ab}}$. Now

$$
\Gamma_{G^{\mathrm{Ab}}}\left(\left(\Gamma_{2}(G)\right)^{\mathrm{Ab}}\right)=\left[G, \Gamma_{2}(G)\right] /\left[\Gamma_{2}(G), \Gamma_{2}(G)\right]=\Gamma_{3}(G) /\left[\Gamma_{2}(G), \Gamma_{2}(G)\right],
$$

and since $\Gamma_{3}(G) \subseteq \Gamma_{2}(G)$, it follows that $\Gamma_{3}(G)=\Gamma_{2}(G)$, which implies the result.

Remark 2.5. The hypothesis of part (2) of the lemma holds for example if $G^{\mathrm{Ab}}$ is cyclic. Recall that if $G^{\mathrm{Ab}}$ is finitely generated, then this condition is also necessary: if $H$ is a finitely-generated Abelian group satisfying $H_{2}(H, \mathbb{Z})=\{0\}$, then $H$ is cyclic.

2.2. The lower central series of $B_{n}\left(\mathbb{S}^{2}\right)$. Now we come to the main result of this section.

Theorem 1.3. For all $n \geq 2$, the lower central series of $B_{n}\left(\mathbb{S}^{2}\right)$ is constant from the commutator subgroup onwards: $\Gamma_{m}\left(B_{n}\left(\mathbb{S}^{2}\right)\right)=\Gamma_{2}\left(B_{n}\left(\mathbb{S}^{2}\right)\right)$ for all $m \geq 2$. The subgroup $\Gamma_{2}\left(B_{n}\left(\mathbb{S}^{2}\right)\right)$ is as follows:

(1) If $n=1,2$, then $\Gamma_{2}\left(B_{n}\left(\mathbb{S}^{2}\right)\right)=\{1\}$.

(2) If $n=3$, then $\Gamma_{2}\left(B_{n}\left(\mathbb{S}^{2}\right)\right) \cong \mathbb{Z}_{3}$. Thus $B_{3}\left(\mathbb{S}^{2}\right) \cong \mathbb{Z}_{3} \rtimes \mathbb{Z}_{4}$, the action being the non-trivial one.

(3) If $n=4$, then $\Gamma_{2}\left(B_{4}\left(\mathbb{S}^{2}\right)\right)$ admits a presentation of the following form:

generators: $g_{1}, g_{2}, g_{3}$, where in terms of the usual generators of $B_{4}\left(\mathbb{S}^{2}\right), g_{1}=$ $\sigma_{1}^{2} \sigma_{2} \sigma_{1}^{-3}, g_{2}=\sigma_{1}^{3} \sigma_{2} \sigma_{1}^{-4}$ and $g_{3}=\sigma_{3} \sigma_{1}^{-1}$. 
relations:

$$
\begin{gathered}
g_{3}^{4}=1, \\
{\left[g_{3}^{2}, g_{1}\right]=1,} \\
{\left[g_{3}^{2}, g_{2}\right]=1,} \\
{\left[g_{3}, g_{2} g_{1}\right]=1,} \\
g_{1}^{-1} g_{3}^{-1} g_{1}=g_{2} g_{3} g_{2}^{-1}, \\
g_{1}^{-1} g_{3}^{-1} g_{1}=g_{1} g_{3} g_{1}^{-1} g_{3} .
\end{gathered}
$$

Furthermore,

$$
\Gamma_{2}\left(B_{4}\left(\mathbb{S}^{2}\right)\right) \cong \mathcal{Q}_{8} \rtimes \mathbb{F}_{2}(a, b),
$$

where $\mathcal{Q}_{8}=\left\langle x, y \mid x^{2}=y^{2}, x y x^{-1}=y^{-1}\right\rangle$ is the quaternion group of order 8 , and $\mathbb{F}_{2}(a, b)$ is the free group of rank 2 on two generators a and $b$. The following elements of $B_{4}\left(\mathbb{S}^{2}\right)$ realise these subgroups: $x=g_{3}$, $y=g_{1} g_{3} g_{1}^{-1}, a=g_{1}$ and $b=g_{2}$. The action is given by:

$$
\begin{array}{ll}
\varphi(a)(x)=y, & \varphi(a)(y)=x y, \\
\varphi(b)(x)=y x, & \varphi(b)(y)=x .
\end{array}
$$

(4) If $n \geq 5$, a presentation for $\Gamma_{2}\left(B_{n}\left(\mathbb{S}^{2}\right)\right)$ is given in Proposition 4.1 .

Proof. The first part of the theorem, $\Gamma_{m}\left(B_{n}\left(\mathbb{S}^{2}\right)\right)=\Gamma_{2}\left(B_{n}\left(\mathbb{S}^{2}\right)\right)$ for $m \geq 2$, follows from Lemma 2.4(2) and Remark 2.5.

Now let us consider the rest of the theorem.

(1) If $n=1,2$, then $B_{n}\left(\mathbb{S}^{2}\right) \cong \mathbb{Z}_{n}$, and the result follows easily.

(2) Let $n=3$. Then $B_{3}\left(\mathbb{S}^{2}\right)$ is a ZS-metacyclic group (a group whose Sylow subgroups, commutator subgroup and commutator quotient group are all cyclic) of order 12 [14]. It follows from Proposition 2.1(1) that $\left(B_{3}\left(\mathbb{S}^{2}\right)\right){ }^{\mathrm{Ab}} \cong \mathbb{Z}_{4}$, and hence $\Gamma_{2}\left(B_{3}\left(\mathbb{S}^{2}\right)\right) \cong \mathbb{Z}_{3}$

From Proposition 2.1(2), the short exact sequence (2.1) splits, so we have $B_{3}\left(\mathbb{S}^{2}\right)$ $\cong \mathbb{Z}_{3} \rtimes \mathbb{Z}_{4}$, and the action of the generator $\widetilde{\sigma}$ of $\left(B_{3}\left(\mathbb{S}^{2}\right)\right)$ Ab on the generator $\rho$ of $\mathbb{Z}_{3}$ is given by $\widetilde{\sigma} \cdot \rho=\rho^{-1}$, i.e. the non-trivial action.

(3) Let $n=4$. To obtain the given presentation of $\Gamma_{2}\left(B_{4}\left(\mathbb{S}^{2}\right)\right)$, one applies the Reidemeister-Schreier rewriting process to the short exact sequence (2.1). The calculations are deferred to Proposition 4.3, see Section 4.2.

Supposing this presentation to be correct, let us prove the second part of (3) of Theorem [1.3] that $\Gamma_{2}\left(B_{4}\left(\mathbb{S}^{2}\right)\right) \cong \mathcal{Q}_{8} \rtimes \mathbb{F}_{2}(a, b)$. Consider the subgroup $H$ of $B_{4}\left(\mathbb{S}^{2}\right)$ generated by $x=g_{3}$ and $y=g_{1} g_{3} g_{1}^{-1}$. By equation (2.6),$x^{2}=g_{3}^{2}=\left(g_{1} g_{3} g_{1}^{-1}\right)^{2}=$ $y^{2}$. Now

$$
\begin{aligned}
y^{-1} & =g_{1} g_{3}^{-1} g_{1}^{-1}=\sigma_{1}^{2} \sigma_{2} \sigma_{1}^{-3} \cdot \sigma_{1} \sigma_{3}^{-1} \cdot \sigma_{1}^{3} \sigma_{2}^{-1} \sigma_{1}^{-2} \\
& =\sigma_{1}^{2} \sigma_{2} \sigma_{1} \sigma_{3} \sigma_{2}=\sigma_{1}^{2} \sigma_{2} \sigma_{3} \sigma_{1} \sigma_{2},
\end{aligned}
$$

and

$$
y^{-1} x^{-1}=\sigma_{1}^{2} \sigma_{2} \sigma_{3} \sigma_{1} \sigma_{2} \sigma_{3}^{-1} \sigma_{1}=\sigma_{1}^{2} \sigma_{2} \sigma_{1} \sigma_{2}^{-1} \sigma_{3} \sigma_{2} \sigma_{1}=\sigma_{1} \sigma_{2} \sigma_{3} \sigma_{1} \sigma_{2} \sigma_{1}
$$

which is the half-twist of $B_{4}\left(\mathbb{S}^{2}\right)$. It was shown in [24] (see the first paragraph of the proof of the Theorem of that paper) that the two elements $y^{-1} x^{-1}$ and $y^{-1}$ together generate a subgroup of $B_{4}\left(\mathbb{S}^{2}\right)$ isomorphic to $\mathcal{Q}_{8}$. Since these two elements generate 
$H$, we conclude that $H$ is isomorphic to $\mathcal{Q}_{8}$. Further, $H$ is normal in $\Gamma_{2}\left(B_{4}\left(\mathbb{S}^{2}\right)\right)$. This is a consequence of the following identities:

$$
\left\{\begin{array}{l}
g_{1} x g_{1}^{-1}=y \\
g_{1} y g_{1}^{-1}=x^{-1} y^{-1}=x y \quad \text { by equation (2.10) } \\
g_{2} x g_{2}^{-1}=g_{1}^{-1} g_{3}^{-1} g_{1}=y x \quad \text { by equations (2.9) and (2.10), } \\
g_{2} y g_{2}^{-1}=x \quad \text { by equation (2.8). }
\end{array}\right.
$$

Since $y=g_{1} g_{3} g_{1}^{-1}$, this normality implies that $H$ is in fact the normal closure of $g_{3}$ in $\Gamma_{2}\left(B_{4}\left(\mathbb{S}^{2}\right)\right)$. Studying the given presentation of $\Gamma_{2}\left(B_{4}\left(\mathbb{S}^{2}\right)\right)$, we see that $\Gamma_{2}\left(B_{4}\left(\mathbb{S}^{2}\right)\right) / H$ is isomorphic to the free group $\mathbb{F}_{2}(a, b)$ on two generators $a$ and $b$ (which are the $H$-cosets of $g_{1}$ and $g_{2}$ ). Thus the short exact sequence $1 \longrightarrow H \longrightarrow$ $\Gamma_{2}\left(B_{4}\left(\mathbb{S}^{2}\right)\right) \longrightarrow \mathbb{F}_{2}(a, b) \longrightarrow 1$ splits, a section being given by sending $a$ to $g_{1}$ and $b$ to $g_{2}$. The action is then defined by the equations (2.11), which are those given in the statement of the theorem.

(4) Now suppose that $n \geq 5$. A presentation is given in Proposition 4.1. This completes the proof of Theorem 1.3 .

\section{THE DERIVED SERIES OF $B_{n}\left(\mathbb{S}^{2}\right)$}

In this section, we study the derived series of $B_{n}\left(\mathbb{S}^{2}\right)$. The aim is to prove the following result, which shows that for all $n \neq 3,4,\left(B_{n}\left(\mathbb{S}^{2}\right)\right)^{(1)}$ is perfect. The difficult case is $n=4$, but using the semi-direct product structure of $\left(B_{4}\left(\mathbb{S}^{2}\right)\right)^{(1)}$ obtained in Theorem 1.3 , we shall be able to prove that the derived series of $B_{4}\left(\mathbb{S}^{2}\right)$ coincides from a certain point with that of the free group of rank 2 . Before doing so, we recall the statement of Theorem 1.4, and then state and prove Proposition 3.3 which describes the commutator subgroup of a general semi-direct product.

Theorem 1.4. The derived series of $B_{n}\left(\mathbb{S}^{2}\right)$ is as follows:

(1) If $n=1,2$, then $\left(B_{n}\left(\mathbb{S}^{2}\right)\right)^{(1)}=\{1\}$.

(2) If $n=3$, then $\left(B_{3}\left(\mathbb{S}^{2}\right)\right)^{(1)} \cong \mathbb{Z}_{3}$ and $\left(B_{3}\left(\mathbb{S}^{2}\right)\right)^{(2)}=\{1\}$.

(3) Suppose that $n=4$. Then:

(a) $\left(B_{4}\left(\mathbb{S}^{2}\right)\right)^{(1)}=\Gamma_{2}\left(B_{4}\left(\mathbb{S}^{2}\right)\right)$ is given by part (3) of Theorem 1.3; it is isomorphic to the semi-direct product $\mathcal{Q}_{8} \rtimes \mathbb{F}_{2}$. Further, the quotient $B_{4}\left(\mathbb{S}^{2}\right) /\left(B_{4}\left(\mathbb{S}^{2}\right)\right)^{(1)}$ is isomorphic to $\mathbb{Z}_{6}$.

(b) $\left(B_{4}\left(\mathbb{S}^{2}\right)\right)^{(2)}$ is isomorphic to the semi-direct product $\mathcal{Q}_{8} \rtimes\left(\mathbb{F}_{2}\right)^{(1)}$, where $\left(\mathbb{F}_{2}\right)^{(1)}$ is the commutator subgroup of the free group $\mathbb{F}_{2}(a, b)$ of rank 2 on two generators $a, b$. The action of $\left(\mathbb{F}_{2}\right)^{(1)}$ on $\mathcal{Q}_{8}$ is the restriction of the action of $\mathbb{F}_{2}(a, b)$ given in part (3) of Theorem 1.3 . Further,

$\left(B_{4}\left(\mathbb{S}^{2}\right)\right)^{(1)} /\left(B_{4}\left(\mathbb{S}^{2}\right)\right)^{(2)} \cong \mathbb{Z}^{2}$ and $B_{4}\left(\mathbb{S}^{2}\right) /\left(B_{4}\left(\mathbb{S}^{2}\right)\right)^{(2)} \cong \mathbb{Z}^{2} \rtimes \mathbb{Z}_{6}$,

where the action of the generator $\bar{\sigma}$ of $\mathbb{Z}_{6}$ on $\mathbb{Z}^{2}$ is given by left multiplication by the matrix $\left(\begin{array}{cc}0 & 1 \\ -1 & 1\end{array}\right)$.

(c) $\left(B_{4}\left(\mathbb{S}^{2}\right)\right)^{(3)}$ is a subgroup of $P_{4}\left(\mathbb{S}^{2}\right)$ isomorphic to the direct product $\mathbb{Z}_{2} \times\left(\mathbb{F}_{2}\right)^{(2)}$. Further,

$$
\left(B_{4}\left(\mathbb{S}^{2}\right)\right)^{(2)} /\left(B_{4}\left(\mathbb{S}^{2}\right)\right)^{(3)} \cong\left(\mathbb{Z}_{2} \times \mathbb{Z}_{2}\right) \times\left(\mathbb{F}_{2}\right)^{(1)} /\left(\mathbb{F}_{2}\right)^{(2)} .
$$


(d) $\left(B_{4}\left(\mathbb{S}^{2}\right)\right)^{(m)} \cong\left(\mathbb{F}_{2}\right)^{(m-1)}$ for all $m \geq 4$. Further,

$$
\left(B_{4}\left(\mathbb{S}^{2}\right)\right)^{(3)} /\left(B_{4}\left(\mathbb{S}^{2}\right)\right)^{(4)} \cong \mathbb{Z}_{2} \times\left(\mathbb{F}_{2}\right)^{(2)} /\left(\mathbb{F}_{2}\right)^{(3)},
$$

and for $m \geq 4$,

$$
\left(B_{4}\left(\mathbb{S}^{2}\right)\right)^{(m)} /\left(B_{4}\left(\mathbb{S}^{2}\right)\right)^{(m+1)} \cong\left(\mathbb{F}_{2}\right)^{(m-1)} /\left(\mathbb{F}_{2}\right)^{(m)} .
$$

(4) If $n \geq 5$, then $\left(B_{n}\left(\mathbb{S}^{2}\right)\right)^{(2)}=\left(B_{n}\left(\mathbb{S}^{2}\right)\right)^{(1)}$, so $\left(B_{n}\left(\mathbb{S}^{2}\right)\right)^{(1)}$ is perfect. $A$ presentation of $\left(B_{n}\left(\mathbb{S}^{2}\right)\right)^{(1)}$ is given in Proposition 4.1 .

Remark 3.1. In part (3) of Theorem 1.4 and also in what follows, we shall often refer to the derived series of $\mathbb{F}_{2}(a, b)$ as well as its quotients. We were not able to track down an explicit reference for them, but one may observe that for $i \geq 1$, $\left(\mathbb{F}_{2}(a, b)\right)^{(i)}$ is a free group of infinite rank, and hence $\left(\mathbb{F}_{2}(a, b)\right)^{(i)} /\left(\mathbb{F}_{2}(a, b)\right)^{(i+1)}$ is a free Abelian group of infinite rank. A basis of $\left(\mathbb{F}_{2}(a, b)\right)^{(1)}=\Gamma_{2}\left(\mathbb{F}_{2}(a, b)\right)$ may be obtained as follows: considering the short exact sequence (2.4) with $G=\mathbb{F}_{2}(a, b)$, $\left(\mathbb{F}_{2}(a, b)\right)^{(1)}$ may be identified with the fundamental group of the Cayley graph of $\mathbb{F}_{2}(a, b)$. Let $\mathcal{T}$ be a maximal tree in this graph. For each $g \in \mathbb{F}_{2}(a, b)$, let $w_{g}$ be the word corresponding to the path in $\mathcal{T}$ between $e$ and $g$. Then a basis is given by the set of elements of the form $w_{g}[a, b] w_{g}^{-1}$, where $g$ runs over $\mathbb{F}_{2}(a, b)$. For example, the set $\left\{a^{p} b^{q}[a, b] b^{-q} a^{-p}\right\}_{p, q \in \mathbb{Z}}$ is a basis of $\left(\mathbb{F}_{2}(a, b)\right)^{(1)}$. Since $\mathbb{F}_{2}(a, b)$ is residually nilpotent and $\left(\mathbb{F}_{2}(a, b)\right)^{(i-1)} \subseteq \Gamma_{i}\left(\mathbb{F}_{2}(a, b)\right)$, it follows that $\bigcap_{i \geq 0}\left(\mathbb{F}_{2}(a, b)\right)^{(i)}=\{1\}$ and $\mathbb{F}_{2}(a, b)$ is residually soluble.

We easily obtain the following corollary of Theorem 1.4.

Corollary 3.2. Let $n \in \mathbb{N}$. Then $B_{n}\left(\mathbb{S}^{2}\right)$ is residually soluble if and only if $n \leq 4$.

Proof of Corollary 3.2, Recall that a group $G$ is residually soluble if and only if $\bigcap_{i \geq 0} G^{(i)}=\{1\}$. If $n=1,2,3$, this is obvious, and if $n=4$, the residual solubility of $B_{4}\left(\mathbb{S}^{2}\right)$ follows from that of $\mathbb{F}_{2}(a, b)$. For $n \geq 5$, the result also follows easily, since $\left(B_{n}\left(\mathbb{S}^{2}\right)\right)^{(1)}$ is non-trivial.

Before proving Theorem [.4 let us state and prove the following proposition which describes the commutator subgroup of a semi-direct product. This result will be used frequently throughout the rest of this paper.

Proposition 3.3. Let $G, H$ be groups, and let $\varphi: G \longrightarrow A u t(H)$ be an action of $G$ on $H$. Let $\widehat{H}$ be the subgroup of $H$ generated by the elements of the form $\varphi(g)(h)$. $h^{-1}$, where $g \in G, h \in H$, and let $L$ be the subgroup of $H$ generated by $\Gamma_{2}(H)$ and $\widehat{H}$. Then $\varphi$ induces an action (also denoted by $\varphi$ ) of $\Gamma_{2}(G)$ on $L$, and $L \rtimes_{\varphi} \Gamma_{2}(G)=$ $\Gamma_{2}\left(H \rtimes_{\varphi} G\right)$. In particular, $\Gamma_{2}\left(H \rtimes_{\varphi} G\right)$ is the subgroup generated by $\Gamma_{2}(H), \Gamma_{2}(G)$ and $\widehat{H}$.

Remark 3.4. We claim that $L$ is none other than the commutator subgroup $\Gamma_{G}(H)$ defined by equation (2.2) with respect to the given action. To see this, recall that $\Gamma_{G}(H)$ is the subgroup of $H$ generated by the elements of the form $\varphi(g)(h) \cdot k h^{-1} k^{-1}$, where $g \in G$ and $h, k \in H$. Taking $g=e$ (respectively $k=e$ ), it follows that $\Gamma_{G}(H) \supseteq \Gamma_{2}(H)$ (respectively $\Gamma_{G}(H) \supseteq \widehat{H}$ ), and hence $L \subseteq \Gamma_{G}(H)$. Conversely, $\varphi(g)(h) \cdot k h^{-1} k^{-1}=\varphi(g)(h) h^{-1} \cdot h k h^{-1} k^{-1} \in L$, so $\Gamma_{G}(H) \subseteq L$, and the claim is 
proved. Note further that if $h^{\prime} \in H$, then there exists $h^{\prime \prime} \in H$ such that $\varphi(g)\left(h^{\prime \prime}\right)=$ $h^{\prime}$, so

$$
h^{\prime}\left(\varphi(g)(h) \cdot h^{-1}\right) h^{\prime-1}=\varphi(g)\left(h^{\prime \prime} h\right)\left(h^{\prime \prime} h\right)^{-1} \cdot\left(\varphi(g)\left(h^{\prime \prime}\right) h^{\prime \prime-1}\right)^{-1} .
$$

It follows that $\widehat{H}$ and $L$ are normal in $H$. In particular, $\Gamma_{G}(H)$ is normal in $H$.

Proof of Proposition 3.3. From now on, we shall identify each subgroup $H_{1}$ of $H$ (respectively each subgroup $G_{1}$ of $G$ ) with the corresponding subgroup of the form $\left\{(h, 1) \mid h \in H_{1}\right\}$ (respectively $\left\{(1, g) \mid g \in G_{1}\right\}$ ) of $H \rtimes_{\varphi} G$ without further comment. The group operation in $H \rtimes_{\varphi} G$ shall be written as

$$
(h, g) \star\left(h^{\prime}, g^{\prime}\right)=\left(h . \varphi(g)(h), g g^{\prime}\right) \text {, where }(h, g),\left(h^{\prime}, g^{\prime}\right) \in H \rtimes_{\varphi} G \text {. }
$$

The subgroup $L$ is normal in $H$ by Remark 3.4. Let us show that $\varphi$ induces an action (also denoted by $\varphi$ ) of $G$ on $L$. Let $g \in G$. Since $\varphi(g)\left(\left[h_{1}, h_{2}\right]\right)=$ $\left[\varphi(g)\left(h_{1}\right), \varphi(g)\left(h_{2}\right)\right] \in \Gamma_{2}(H)$ for all $h_{1}, h_{2} \in H$, and

$$
\varphi(g)\left(\varphi\left(g^{\prime}\right)(h) h^{-1}\right)=\varphi\left(g g^{\prime}\right)(h) h^{-1} \cdot\left(\varphi(g)(h) h^{-1}\right)^{-1} \in \widehat{H}
$$

for all $h \in H$ and $g^{\prime} \in G$, it follows that $\varphi(g)(L) \subseteq L$. Clearly $\varphi(g)$ is injective. The surjectivity of $\varphi(g)$ (restricted to $L$ ) may be deduced from the following observations:

(1) if $i=1,2$ and $h_{i}^{\prime} \in H$, then there exists $h_{i} \in H$ such that $\varphi(g)\left(h_{i}\right)=h_{i}^{\prime}$, and hence $\varphi(g)\left(\left[h_{1}, h_{2}\right]\right)=\left[h_{1}^{\prime}, h_{2}^{\prime}\right]$.

(2) if $g^{\prime} \in G$ and $h, h^{\prime} \in H$, then

$$
\varphi(g)\left(\varphi\left(g^{-1} g^{\prime}\right)(h) h^{-1} \cdot h\left(\varphi\left(g^{-1}\right)\left(h^{-1}\right) h\right) h^{-1}\right)=\varphi\left(g^{\prime}\right)(h) h^{-1} .
$$

Thus $\varphi$ induces an action (also denoted by $\varphi$ ) of $\Gamma_{2}(G)$ on $L$, and $L \rtimes_{\varphi} \Gamma_{2}(G)$ is a subgroup of $H \rtimes_{\varphi} G$.

Clearly any element of $\Gamma_{2}(H)$ (respectively $\Gamma_{2}(G)$ ) may be written as an element of $\Gamma_{2}\left(H \rtimes_{\varphi} G\right)$. Further, if $g \in G$ and $h \in H$, then

$$
[(1, g),(h, 1)]=(\varphi(g)(h), 1) \star\left(h^{-1}, 1\right)=\left(\varphi(g)(h) h^{-1}, 1\right),
$$

and thus every element of $\widehat{H}$ may be written as an element of $\Gamma_{2}\left(H \rtimes_{\varphi} G\right)$. This proves that $L \rtimes_{\varphi} \Gamma_{2}(G) \subseteq \Gamma_{2}\left(H \rtimes_{\varphi} G\right)$.

To see the converse, note that the commutator of the elements $\left(h_{1}, g_{1}\right),\left(h_{2}, g_{2}\right) \in$ $H \rtimes_{\varphi} G$ may be written as

$$
\left[\left(h_{1}, g_{1}\right),\left(h_{2}, g_{2}\right)\right]=\left(h_{1} \cdot \varphi\left(g_{1}\right)\left(h_{2}\right) \cdot \varphi\left(g_{1} g_{2} g_{1}^{-1}\right)\left(h_{1}^{-1}\right) \cdot \varphi\left(\left[g_{1}, g_{2}\right]\right)\left(h_{2}^{-1}\right),\left[g_{1}, g_{2}\right]\right) .
$$

The second factor belongs to $\Gamma_{2}(G)$. The first factor is of the form

$$
\begin{aligned}
& {\left[h_{1}, h_{2}\right] \cdot h_{2} h_{1} h_{2}^{-1}\left(\varphi\left(g_{1}\right)\left(h_{2}\right) h_{2}^{-1}\right) h_{2} h_{1}^{-1} h_{2}^{-1}} \\
& \cdot h_{2} h_{1}\left(\varphi\left(g_{1} g_{2} g_{1}^{-1}\right)\left(h_{1}^{-1}\right) h_{1}\right) h_{1}^{-1} h_{2}^{-1} \cdot h_{2}\left(\varphi\left(\left[g_{1}, g_{2}\right]\right)\left(h_{2}^{-1}\right) h_{2}\right) h_{2}^{-1},
\end{aligned}
$$

which is a product of elements of $L$. Hence $\Gamma_{2}\left(H \rtimes_{\varphi} G\right) \subseteq L \rtimes_{\varphi} \Gamma_{2}(G)$, and the proposition follows.

We now prove the main result of this section.

Proof of Theorem 1.4. Cases (11) and (2) follow directly from Theorem 1.3 . 
Now consider case (4), i.e. $n \geq 5$. Let $H \subseteq\left(B_{n}\left(\mathbb{S}^{2}\right)\right)^{(1)}$ be a normal subgroup of $B_{n}\left(\mathbb{S}^{2}\right)$ such that $A=\left(B_{n}\left(\mathbb{S}^{2}\right)\right)^{(1)} / H$ is Abelian (notice that this condition is satisfied if $\left.H=\left(B_{n}\left(\mathbb{S}^{2}\right)\right)^{(2)}\right)$. Let

$$
\left\{\begin{aligned}
\pi: B_{n}\left(\mathbb{S}^{2}\right) & \longrightarrow B_{n}\left(\mathbb{S}^{2}\right) / H, \\
\beta & \longmapsto \bar{\beta}
\end{aligned}\right.
$$

denote the canonical projection. Then the Abelianisation homomorphism

$$
\alpha: B_{n}\left(\mathbb{S}^{2}\right) \longrightarrow\left(B_{n}\left(\mathbb{S}^{2}\right)\right)^{\mathrm{Ab}}
$$

of Section 2 factors through $B_{n}\left(\mathbb{S}^{2}\right) / H$, i.e. there exists a (surjective) homomorphism $\widehat{\alpha}: B_{n}\left(\mathbb{S}^{2}\right) / H \longrightarrow\left(B_{n}\left(\mathbb{S}^{2}\right)\right){ }^{\mathrm{Ab}}$ satisfying $\alpha=\widehat{\alpha} \circ \pi$. So we have the following short exact sequence:

$$
1 \longrightarrow A \longrightarrow B_{n}\left(\mathbb{S}^{2}\right) / H \stackrel{\widehat{\alpha}}{\longrightarrow}\left(B_{n}\left(\mathbb{S}^{2}\right)\right)^{\mathrm{Ab}} \longrightarrow 1 .
$$

Now $\overline{\sigma_{1}}, \ldots, \overline{\sigma_{n-1}}$ generate $B_{n}\left(\mathbb{S}^{2}\right) / H$, but since $\alpha\left(\sigma_{i}\right)=\alpha\left(\sigma_{1}\right)$ for $1 \leq i \leq n-1$, it follows that $\widehat{\alpha}\left(\overline{\sigma_{i}}\right)=\widehat{\alpha}\left(\overline{\sigma_{1}}\right)$, and so there exists $t_{i} \in A$ such that $\overline{\sigma_{i}}=t_{i} \overline{\sigma_{1}}$.

We now apply $\pi$ to each of the relations of equation (1.2) of $B_{n}\left(\mathbb{S}^{2}\right)$. First suppose that $3 \leq i \leq n-1$. Since $\sigma_{i}$ commutes with $\sigma_{1}$, we have that $\overline{\sigma_{1}} \cdot t_{i} \overline{\sigma_{1}}=t_{i} \overline{\sigma_{1}} \cdot \overline{\sigma_{1}}$, and hence $t_{i}$ commutes with $\overline{\sigma_{1}}$.

Now let $4 \leq i \leq n-1$. Since $\sigma_{i}$ commutes with $\sigma_{2}$, we obtain $t_{i} \overline{\sigma_{1}} \cdot t_{2} \overline{\sigma_{1}}=$ $t_{2} \overline{\sigma_{1}} \cdot t_{i} \overline{\sigma_{1}}$. Since $A$ is Abelian, it follows from the previous paragraph that $t_{2}$ commutes with $\overline{\sigma_{1}}$. Applying this to the image of the relation $\sigma_{1} \sigma_{2} \sigma_{1}=\sigma_{2} \sigma_{1} \sigma_{2}$, under $\pi$, we see that $t_{2}=t_{2}^{2}$, and hence $t_{2}=1$.

Next, if $i \geq 2$, then the relation $\sigma_{i} \sigma_{i+1} \sigma_{i}=\sigma_{i+1} \sigma_{i} \sigma_{i+1}$ implies that $t_{i}=t_{i+1}$, and so $t_{2}=\cdots=t_{n-1}=1$. Hence $\overline{\sigma_{1}}=\overline{\sigma_{2}}=\cdots=\overline{\sigma_{n-1}}$. Thus $B_{n}\left(\mathbb{S}^{2}\right) / H$ is cyclic, generated by $\overline{\sigma_{1}}$, and finite of order not greater than $2(n-1)$, because the surface relation $\sigma_{1} \cdots \sigma_{n-2} \sigma_{n-1}^{2} \sigma_{n-2} \cdots \sigma_{1}=1$ projects to ${\overline{\sigma_{1}}}^{2(n-1)}=1$. Since $\widehat{\alpha}$ is surjective and $\left(B_{n}\left(\mathbb{S}^{2}\right)\right) \mathrm{Ab} \cong \mathbb{Z}_{2(n-1)}$, we conclude that $\widehat{\alpha}$ is an isomorphism, so $B_{n}\left(\mathbb{S}^{2}\right) / H \cong \mathbb{Z}_{2(n-1)}$, and $A=\left(B_{n}\left(\mathbb{S}^{2}\right)\right)^{(1)} / H$ is trivial. In particular

$$
\left(B_{n}\left(\mathbb{S}^{2}\right)\right)^{(2)}=\left[\left(B_{n}\left(\mathbb{S}^{2}\right)\right)^{(1)},\left(B_{n}\left(\mathbb{S}^{2}\right)\right)^{(1)}\right]=\left(B_{n}\left(\mathbb{S}^{2}\right)\right)^{(1)},
$$

so $\left(B_{n}\left(\mathbb{S}^{2}\right)\right)^{(1)}$ is perfect.

Now consider case (3), so $n=4$. Recall that part (3a) was proved in Theorem 1.3 and Proposition 2.1. To obtain $\left(B_{4}\left(\mathbb{S}^{2}\right)\right)^{(2)}$, it suffices to observe that for the action of $\mathbb{F}_{2}(a, b)$ on $\mathcal{Q}_{8}$, the subgroup $\widehat{\mathcal{Q}_{8}}$ defined in Proposition 3.3 is $\mathcal{Q}_{8}$ (which is the case, since by Theorem 1.3(3), $\varphi(b)(x) x^{-1}=y$ and $\left.\varphi(a)(y) y^{-1}=x\right)$. So $\left(B_{4}\left(\mathbb{S}^{2}\right)\right)^{(2)}$ is generated by $\mathcal{Q}_{8}$ and $\left(\mathbb{F}_{2}\right)^{(1)},\left(B_{4}\left(\mathbb{S}^{2}\right)\right)^{(2)} \cong \mathcal{Q}_{8} \rtimes\left(\mathbb{F}_{2}\right)^{(1)}$, and the action is the restriction of that of $\mathbb{F}_{2}(a, b)$ on $\mathcal{Q}_{8}$, which proves the first part of (3b).

To determine $\left(B_{4}\left(\mathbb{S}^{2}\right)\right)^{(3)}$, we first have to describe the subgroup $\widehat{\mathcal{Q}_{8}}$ for the action of $\left(\mathbb{F}_{2}\right)^{(1)}$ on $\mathcal{Q}_{8}$. By Theorem [1.3)(3), if $B=[a, b] \in\left(\mathbb{F}_{2}(a, b)\right)^{(1)}$, then the automorphism $\varphi(B)$ satisifies $\varphi(B)(z)=x^{2} \cdot z$ for $z \in\{x, y\}$ (recall that $x^{2}=y^{2}$ ). Since $\left(\mathbb{F}_{2}(a, b)\right)^{(1)}$ is the subgroup of $\mathbb{F}_{2}(a, b)$ normally generated by $B$ and the centre $\left\langle x^{2}\right\rangle$ of $\mathcal{Q}_{8}$ is invariant under $\operatorname{Aut}\left(\mathcal{Q}_{8}\right)$, it follows that $\widehat{\mathcal{Q}_{8}}=\left\langle x^{2}\right\rangle$. So $\left(B_{4}\left(\mathbb{S}^{2}\right)\right)^{(3)}$ is isomorphic to the semi-direct product of $\mathbb{Z}_{2}$ by $\left(\mathbb{F}_{2}\right)^{(2)}$. But the action is trivial, and so the product is direct. This proves the first part of (3C).

For $m \geq 4$, the subgroup $\left(B_{4}\left(\mathbb{S}^{2}\right)\right)^{(m)}$ is clear from the description of $\left(B_{4}\left(\mathbb{S}^{2}\right)\right)^{(3)}$, and hence we obtain the first part of $(3 \mathrm{~d})$. 
For several values of $m$, we now analyse the quotients $B_{4}\left(\mathbb{S}^{2}\right) /\left(B_{4}\left(\mathbb{S}^{2}\right)\right)^{(m)}$ and $\left(B_{4}\left(\mathbb{S}^{2}\right)\right)^{(m-1)} /\left(B_{4}\left(\mathbb{S}^{2}\right)\right)^{(m)}$. For the quotient $B_{4}\left(\mathbb{S}^{2}\right) /\left(B_{4}\left(\mathbb{S}^{2}\right)\right)^{(m)}$, we shall consider the case $m=2$ (the case $m=1$ is given by Proposition 2.1(1)). For $\left(B_{4}\left(\mathbb{S}^{2}\right)\right)^{(m-1)} /\left(B_{4}\left(\mathbb{S}^{2}\right)\right)^{(m)}$, we consider the cases $m \geq 2$ (the case $m=1$ was considered in Proposition 2.1(10). If $m>4$, the problem reduces to the corresponding problem for the free group on two generators.

We adopt the notation used above in the case $n \geq 5$, and again we suppose that $H \subseteq\left(B_{n}\left(\mathbb{S}^{2}\right)\right)^{(1)}$ is a normal subgroup of $B_{n}\left(\mathbb{S}^{2}\right)$ such that $A=\left(B_{n}\left(\mathbb{S}^{2}\right)\right)^{(1)} / H$ is Abelian. So we have a short exact sequence:

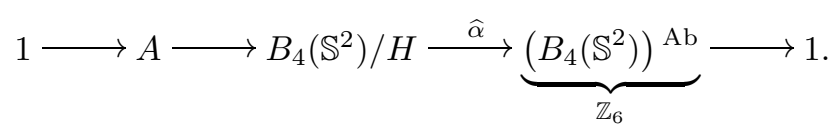

Now $\overline{\sigma_{1}}, \overline{\sigma_{2}}, \overline{\sigma_{3}}$ generate $B_{4}\left(\mathbb{S}^{2}\right) / H$. As above, for $i=2,3$ we set $\overline{\sigma_{i}}=t_{i} \overline{\sigma_{1}}$, where $t_{i} \in A$, and we apply $\pi$ to the relations of $B_{4}\left(\mathbb{S}^{2}\right)$. The fact that $\sigma_{1}$ commutes with $\sigma_{3}$ implies that $t_{3}$ commutes with $\overline{\sigma_{1}}$. The relation $\sigma_{1} \sigma_{2} \sigma_{1}=\sigma_{2} \sigma_{1} \sigma_{2}$ implies that

$$
\bar{\sigma}_{1} t_{2}{\overline{\sigma_{1}}}^{-1}=t_{2} \cdot{\overline{\sigma_{1}}}^{2} t_{2}{\overline{\sigma_{1}}}^{-2} .
$$

Similarly the relation $\sigma_{2} \sigma_{3} \sigma_{2}=\sigma_{3} \sigma_{2} \sigma_{3}$ implies that $t_{2}{\overline{\sigma_{1}}}^{2} t_{2}=t_{3} \overline{\sigma_{1}} t_{2} \overline{\sigma_{1}}$, since $A$ is Abelian and $t_{3}$ commutes with $\overline{\sigma_{1}}$. Thus $t_{2}{\overline{\sigma_{1}}}^{2} t_{2}=t_{3} t_{2}{\overline{\sigma_{1}}}^{2} t_{2}$ from equation (3.1), and so $t_{3}=1$. We conclude that $B_{4}\left(\mathbb{S}^{2}\right) / H$ is generated by $\overline{\sigma_{1}}$ and $t_{2} \overline{\sigma_{1}}$.

Finally, we consider the image of the surface relation under $\pi$. Using equation (3.1), note first that

$$
\begin{aligned}
{\overline{\sigma_{1}}}^{3} t_{2}{\overline{\sigma_{1}}}^{-3} & =\overline{\sigma_{1}}\left(t_{2}^{-1} \cdot{\overline{\sigma_{1}}}_{2}{\overline{\sigma_{1}}}^{-1}\right){\overline{\sigma_{1}}}^{-1}={\overline{\sigma_{1}}}_{2}^{-1}{\overline{\sigma_{1}}}^{-1} \cdot{\overline{\sigma_{1}}}^{2} t_{2}{\overline{\sigma_{1}}}^{-2} \\
& =\bar{\sigma}_{1} t_{2}^{-1}{\overline{\sigma_{1}}}^{-1} \cdot t_{2}^{-1}{\overline{\sigma_{1}}}_{2}{\overline{\sigma_{1}}}^{-1}=t_{2}^{-1}
\end{aligned}
$$

since $A$ is normal and Abelian. Thus $\sigma_{1} \sigma_{2} \sigma_{3}^{2} \sigma_{2} \sigma_{1}=1$ implies that

$$
\begin{aligned}
1 & =\overline{\sigma_{1}} \cdot t_{2}{\overline{\sigma_{1}}} \cdot{\overline{\sigma_{1}}}^{2} \cdot t_{2} \overline{\sigma_{1}} \cdot{\overline{\sigma_{1}}}={\overline{\sigma_{1}}}_{2}{\overline{\sigma_{1}}}^{-1} \cdot{\overline{\sigma_{1}}}\left({\overline{\sigma_{1}}}^{3} t_{2}{\overline{\sigma_{1}}}^{-3}\right){\overline{\sigma_{1}}}^{-1} \cdot{\overline{\sigma_{1}}}^{6} \\
& =\bar{\sigma}_{1} t_{2}{\overline{\sigma_{1}}}^{-1} \cdot \bar{\sigma}_{1} t_{2}^{-1}{\overline{\sigma_{1}}}^{-1} \cdot{\overline{\sigma_{1}}}^{6}={\overline{\sigma_{1}}}^{6}
\end{aligned}
$$

from equation (3.2).

Recall that $\Gamma_{2}\left(B_{4}\left(\mathbb{S}^{2}\right)\right)$ is the normal subgroup of $B_{4}\left(\mathbb{S}^{2}\right)$ generated by the commutators of the generators of $B_{4}\left(\mathbb{S}^{2}\right)$. Hence $A$ is the normal subgroup of $B_{4}\left(\mathbb{S}^{2}\right) / H$ generated by $\left[\overline{\sigma_{1}}, t_{2} \overline{\sigma_{1}}\right]=\bar{\sigma}_{1} t_{2} \bar{\sigma}_{1}-1 \cdot t_{2}^{-1}$. Since $A$ is Abelian and $t_{2} \in A$, the action of conjugation on $A$ by $t_{2}$ is trivial. From equation (3.2), the action of $\bar{\sigma}_{1}^{3}$ on $t_{2}$ yields $t_{2}^{-1}$. Further,

$$
\overline{\sigma_{1}}\left(\bar{\sigma}_{1} t_{2}{\overline{\sigma_{1}}}^{-1} t_{2}^{-1}\right){\overline{\sigma_{1}}}^{-1}={\overline{\sigma_{1}}}^{2} t_{2}{\overline{\sigma_{1}}}^{-2} \cdot{\overline{\sigma_{1}}}_{2}^{-1}{\overline{\sigma_{1}}}^{-1}=t_{2}^{-1}
$$

from equation (3.1), and since ${\overline{\sigma_{1}}}^{2}\left(\bar{\sigma}_{1} t_{2}{\overline{\sigma_{1}}}^{-1} t_{2}^{-1}\right){\overline{\sigma_{1}}}^{-2}=\bar{\sigma}_{1} t_{2}^{-1}{\overline{\sigma_{1}}}^{-1}$, it follows that $A$ is the Abelian group generated by $\bar{\sigma}_{1} t_{2}{\overline{\sigma_{1}}}^{-1} t_{2}^{-1}, t_{2}$ and $\overline{\sigma_{1}} t_{2}{\overline{\sigma_{1}}}^{-1}$, and thus by $t_{2}$ and $\overline{\sigma_{1}} t_{2}{\overline{\sigma_{1}}}^{-1}$.

Let $\widetilde{\sigma}=\alpha\left(\sigma_{1}\right)$ denote the generator of $\left(B_{4}\left(\mathbb{S}^{2}\right)\right)$ Ab. Let $M=\left(\begin{array}{cc}0 & 1 \\ -1 & 1\end{array}\right)$; notice that $M$ is of order 6 . We now let $\left(B_{4}\left(\mathbb{S}^{2}\right)\right){ }^{\mathrm{Ab}} \cong \mathbb{Z}_{6}$ act on $\mathbb{Z}^{2}$ as follows:

$$
\tilde{\sigma} \cdot\left(\begin{array}{l}
X_{1} \\
X_{2}
\end{array}\right)=M\left(\begin{array}{l}
X_{1} \\
X_{2}
\end{array}\right)=\left(\begin{array}{c}
X_{2} \\
X_{2}-X_{1}
\end{array}\right)
$$


and so we may form the associated semi-direct product $\mathbb{Z}^{2} \rtimes \mathbb{Z}_{6}$. We now consider the following homomorphism:

$$
\begin{aligned}
& \psi: B_{4}\left(\mathbb{S}^{2}\right) \longrightarrow \mathbb{Z}^{2} \rtimes \mathbb{Z}_{6}, \\
& \sigma_{1}, \sigma_{3} \longmapsto\left(\left(\begin{array}{c}
0 \\
0
\end{array}\right), \widetilde{\sigma}\right), \\
& \sigma_{2} \longmapsto\left(\left(\begin{array}{l}
1 \\
0
\end{array}\right), \tilde{\sigma}\right) .
\end{aligned}
$$

An easy calculation shows that $\psi$ preserves the relations of $B_{4}\left(\mathbb{S}^{2}\right)$, and so is a well-defined homomorphism. Further, since $\psi\left(\sigma_{1}\right)=\left(\left(\begin{array}{l}0 \\ 0\end{array}\right), \widetilde{\sigma}\right), \psi\left(\sigma_{2} \sigma_{1}^{-1}\right)=$ $\left(\left(\begin{array}{l}1 \\ 0\end{array}\right), 1\right)$ and $\psi\left(\left[\sigma_{1}^{-1}, \sigma_{2}\right]\right)=\left(\left(\begin{array}{l}0 \\ 1\end{array}\right), 1\right), \psi$ is surjective.

Now let $H=\left(B_{4}\left(\mathbb{S}^{2}\right)\right)^{(2)}$, and let $\delta: \mathbb{Z}^{2} \rtimes \mathbb{Z}_{6} \longrightarrow \mathbb{Z}_{6}$ denote the projection onto the second factor. Since $\mathbb{Z}_{6}$ is Abelian, it follows that $\delta(\psi(x))$ is trivial for all $x \in\left(B_{4}\left(\mathbb{S}^{2}\right)\right)^{(1)}$, so $\psi(x)$ belongs to the $\mathbb{Z}^{2}$-factor. Hence,

$$
H=\left[\left(B_{4}\left(\mathbb{S}^{2}\right)\right)^{(1)},\left(B_{4}\left(\mathbb{S}^{2}\right)\right)^{(1)}\right] \subseteq \operatorname{ker} \psi,
$$

and thus $\psi$ factors through $A=B_{4}\left(\mathbb{S}^{2}\right) / H$, inducing a (surjective) homomorphism $\widehat{\psi}: B_{4}\left(\mathbb{S}^{2}\right) / H \longrightarrow \mathbb{Z}^{2} \rtimes \mathbb{Z}_{6}$. From the following commutative diagram of short exact sequences,

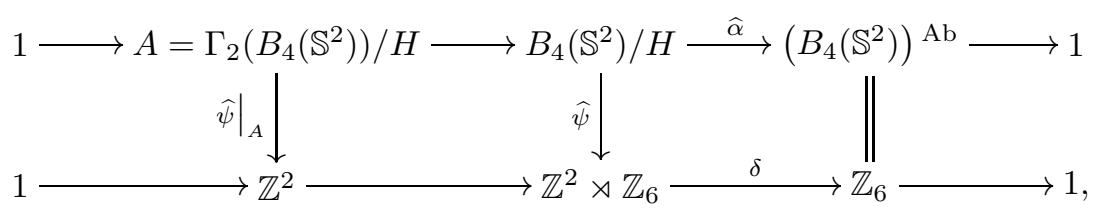

the surjectivity of $\widehat{\psi}$ implies that of $\left.\widehat{\psi}\right|_{A}: A \longrightarrow \mathbb{Z}^{2}$. But $A$ is an Abelian group generated by $\left\{t_{2}, \bar{\sigma} t_{2} \bar{\sigma}^{-1}\right\}$, so $\left.\widehat{\psi}\right|_{A}$ is an isomorphism, and by the 5-Lemma, $\widehat{\psi}$ is too. Hence,

$$
\left(B_{4}\left(\mathbb{S}^{2}\right)\right)^{(1)} /\left(B_{4}\left(\mathbb{S}^{2}\right)\right)^{(2)} \cong \mathbb{Z}^{2} \quad \text { and } \quad B_{4}\left(\mathbb{S}^{2}\right) /\left(B_{4}\left(\mathbb{S}^{2}\right)\right)^{(2)} \cong \mathbb{Z}^{2} \rtimes \mathbb{Z}_{6}
$$

In fact the first of these two equations may be obtained directly since we know that $\left(B_{4}\left(\mathbb{S}^{2}\right)\right)^{(1)} \cong \mathcal{Q}_{8} \rtimes \mathbb{F}_{2}$, and $\left(B_{4}\left(\mathbb{S}^{2}\right)\right)^{(2)}$ is isomorphic to the subgroup $\mathcal{Q}_{8} \rtimes$ $\left(\mathbb{F}_{2}\right)^{(1)}$ of $\mathcal{Q}_{8} \rtimes \mathbb{F}_{2}$, so $\left(B_{4}\left(\mathbb{S}^{2}\right)\right)^{(1)} /\left(B_{4}\left(\mathbb{S}^{2}\right)\right)^{(2)} \cong \mathbb{F}_{2} /\left(\mathbb{F}_{2}\right)^{(1)} \cong \mathbb{Z}^{2}$. Similarly, $\left(B_{4}\left(\mathbb{S}^{2}\right)\right)^{(2)} /\left(B_{4}\left(\mathbb{S}^{2}\right)\right)^{(3)} \cong\left(\mathbb{Z}_{2} \times \mathbb{Z}_{2}\right) \times\left(\mathbb{F}_{2}\right)^{(1)} /\left(\mathbb{F}_{2}\right)^{(2)},\left(B_{4}\left(\mathbb{S}^{2}\right)\right)^{(3)} /\left(B_{4}\left(\mathbb{S}^{2}\right)\right)^{(4)} \cong$ $\mathbb{Z}_{2} \times\left(\mathbb{F}_{2}\right)^{(2)} /\left(\mathbb{F}_{2}\right)^{(3)}$, and for $m \geq 4$,

$$
\left(B_{4}\left(\mathbb{S}^{2}\right)\right)^{(m)} /\left(B_{4}\left(\mathbb{S}^{2}\right)\right)^{(m+1)} \cong\left(\mathbb{F}_{2}\right)^{(m-1)} /\left(\mathbb{F}_{2}\right)^{(m)} .
$$

This proves the remaining parts of (3), and thus completes the proof of Theorem 1.4

\section{Presentations For $\Gamma_{2}\left(B_{n}\left(\mathbb{S}^{2}\right)\right), n \geq 4$}

In Section 4.1, we derive a general presentation obtained using the ReidemeisterSchreier rewriting process. In Section 4.2, we consider the case $n=4$, and obtain the presentation given in Theorem $1.3(3)$. 


\subsection{A general presentation of $\Gamma_{2}\left(B_{n}\left(\mathbb{S}^{2}\right)\right)$ for $n \geq 4$.}

Proposition 4.1. Let $n \geq 4$. The following constitutes a presentation of the group $\Gamma_{2}\left(B_{n}\left(\mathbb{S}^{2}\right)\right)$ :

generators:

$$
\begin{gathered}
w=\sigma_{1}^{2 n-2}, \\
u_{1}=\sigma_{2} \sigma_{1}^{-1}, u_{2}=\sigma_{1} \sigma_{2} \sigma_{1}^{-2}, \ldots, u_{2 n-2}=\sigma_{1}^{2 n-3} \sigma_{2} \sigma_{1}^{-(2 n-2)}, \\
v_{1}=\sigma_{3} \sigma_{1}^{-1}, \ldots, v_{n-3}=\sigma_{n-1} \sigma_{1}^{-1} .
\end{gathered}
$$

relations:

$$
\begin{gathered}
v_{i} v_{j}=v_{j} v_{i} \text { if }|i-j| \geq 2 \text { and } 1 \leq i, j \leq n-3, \\
v_{i} v_{i+1} v_{i}=v_{i+1} v_{i} v_{i+1} \text { for all } 1 \leq i \leq n-4, \\
w \rightleftharpoons v_{i}, \\
u_{i} v_{j} u_{i+1}^{-1} v_{j}^{-1}=1 \text { for } j \geq 2 \text { and } i=1, \ldots, 2 n-3, \\
u_{2 n-2} v_{j} w u_{1}^{-1} w^{-1} v_{j}^{-1}=1 \text { for } 2 \leq j \leq n-3, \\
u_{i} v_{1} u_{i+2} v_{1}^{-1} u_{i+1}^{-1} v_{1}^{-1}=1 \text { for } i=1, \ldots, 2 n-4, \\
u_{2 n-3} v_{1} w u_{1} w^{-1} v_{1}^{-1} u_{2 n-2}^{-1} v_{1}^{-1}=1, \\
u_{2 n-2} v_{1} w u_{2} v_{1}^{-1} u_{1}^{-1} w^{-1} v_{1}^{-1}=1, \\
u_{i+1} u_{i+2}^{-1} u_{i}^{-1}=1 \text { for all } i=1, \ldots, 2 n-4, \\
u_{2 n-2} w u_{1}^{-1} w^{-1} u_{2 n-3}^{-1}=1, \\
w u_{1} u_{2}^{-1} w^{-1} u_{2 n-2}^{-1}=1, \\
u_{2}\left(v_{1} \cdots v_{n-4} v_{n-3}^{2} v_{n-4} \cdots v_{1}\right) u_{2 n-3} w=1, \\
u_{3}\left(v_{1} \cdots v_{n-4} v_{n-3}^{2} v_{n-4} \cdots v_{1}\right) u_{2 n-2} w=1, \\
u_{i}\left(v_{1} \cdots v_{n-4} v_{n-3}^{2} v_{n-4} \cdots v_{1}\right) w u_{i-3}=1 \text { for } i=4, \ldots, 2 n-2, \\
u_{1}\left(v_{1} \cdots v_{n-4} v_{n-3}^{2} v_{n-4} \cdots v_{1}\right) u_{2 n-4} w=1 .
\end{gathered}
$$

Remark 4.2. The above presentation may be simplified, notably in the cases where $n=4,5$, and may be used to show that $\Gamma_{2}\left(B_{n}\left(\mathbb{S}^{2}\right)\right)$ is perfect for $n \geq 5$.

In what follows, we shall denote by equation $\left(m_{i}\right)$ the equation $(m)$ of the above system for the parameter value $i$.

Proof. Taking the presentation (1.2) of $B_{n}\left(\mathbb{S}^{2}\right)$ and the set $\left\{1, \sigma_{1}, \sigma_{1}^{2}, \ldots, \sigma_{1}^{2 n-3}\right\}$ as a Schreier transversal, we apply the Reidemeister-Schreier rewriting process to the following short exact sequence:

$$
1 \longrightarrow \Gamma_{2}\left(B_{n}\left(\mathbb{S}^{2}\right)\right) \longrightarrow B_{n}\left(\mathbb{S}^{2}\right) \longrightarrow\left(B_{n}\left(\mathbb{S}^{2}\right)\right)^{\mathrm{Ab}} \longrightarrow 1 .
$$

As generators of $\Gamma_{2}\left(B_{n}\left(\mathbb{S}^{2}\right)\right)$, we obtain $w=\sigma_{1}^{2 n-2}, \sigma_{1}^{j} \sigma_{i} \sigma_{1}^{-(j+1)}$ and $\sigma_{1}^{2 n-3} \sigma_{i}$, where $2 \leq i \leq n-1$ and $0 \leq j \leq 2 n-4$. We replace the latter by $\sigma_{1}^{2 n-3} \sigma_{i} \cdot w^{-1}=$ $\sigma_{1}^{2 n-3} \sigma_{i} \sigma_{1}^{-(2 n-2)}$. Now turning to the relations, if $i \geq 3$, then for $j=0, \ldots, 2 n-4$, the relator $\sigma_{1} \sigma_{i} \sigma_{1}^{-1} \sigma_{i}^{-1}$ of $B_{n}\left(\mathbb{S}^{2}\right)$ gives rise to relators

$$
\sigma_{1}^{j} \sigma_{1} \sigma_{i} \sigma_{1}^{-1} \sigma_{i}^{-1} \sigma_{1}^{-j}=\sigma_{1}^{j+1} \sigma_{i} \sigma_{1}^{-(j+2)} \cdot \sigma_{1}^{j+1} \sigma_{i}^{-1} \sigma_{1}^{-j}
$$


of $\Gamma_{2}\left(B_{n}\left(\mathbb{S}^{2}\right)\right)$, so

$$
\sigma_{1}^{j+1} \sigma_{i} \sigma_{1}^{-(j+2)}=\sigma_{1}^{j} \sigma_{i} \sigma_{1}^{-(j+1)}=\sigma_{i} \sigma_{1}^{-1}=v_{i-2} .
$$

If $j=2 n-3$, then we have a relator of the form

$$
\sigma_{1}^{2 n-3} \sigma_{1} \sigma_{i} \sigma_{1}^{-1} \sigma_{i}^{-1} \sigma_{1}^{-(2 n-3)}=\sigma_{1}^{2 n-2} \cdot \sigma_{i} \sigma_{1}^{-1} \cdot \sigma_{1}^{2 n-2} \sigma_{1}^{-(2 n-2)} \sigma_{i}^{-1} \sigma_{1}^{-(2 n-3)},
$$

and thus $v_{i-2}$ commutes with $w$, which gives relation (4.3). If $1 \leq i, j \leq n-3$ and $|i-j| \geq 2$, then the relator $\sigma_{i+2} \sigma_{j+2} \sigma_{i+2}^{-1} \sigma_{j+2}^{-1}$ gives rise to the single relator $v_{i} v_{j} v_{i}^{-1} v_{j}^{-1}$, while if $1 \leq i \leq n-4$, the relator $\sigma_{i+2} \sigma_{i+3} \sigma_{i+2} \sigma_{i+3}^{-1} \sigma_{i+2}^{-1} \sigma_{i+3}^{-1}$ yields the single relator $v_{i} v_{i+1} v_{i}=v_{i+1} v_{i} v_{i+1}$; thus we obtain equations (4.1) and (4.2).

Now for $i=1, \ldots, 2 n-2$, let $u_{i}=\sigma_{1}^{i-1} \sigma_{2} \sigma_{1}^{-i}$. From the relator

$$
\sigma_{1}^{j-1} \sigma_{2} \sigma_{1} \sigma_{2} \sigma_{1}^{-1} \sigma_{2}^{-1} \sigma_{1}^{-1} \sigma_{1}^{-(j-1)} \text {, }
$$

we obtain the relators

$$
\begin{aligned}
& u_{j} u_{j+2} u_{j+1}^{-1} \quad \text { if } j=1, \ldots, 2 n-4, \\
& u_{2 n-3} w u_{1} w^{-1} u_{2 n-2}^{-1} \quad \text { if } j=2 n-3, \text { and } \\
& u_{2 n-2} w u_{2} u_{1}^{-1} w^{-1} \quad \text { if } j=2 n-2,
\end{aligned}
$$

which yield respectively equations (4.9), (4.10) and (4.11).

If $2 \leq i \leq n-3$, then the relator $\sigma_{1}^{j-1} \sigma_{i+2} \sigma_{2} \sigma_{i+2}^{-1} \sigma_{2}^{-1} \sigma_{1}^{-(j-1)}$ yields the relators $v_{i} u_{j+1} v_{i}^{-1} u_{j}^{-1}$ if $j=1, \ldots, 2 n-3$ and $v_{i} w u_{1} w^{-1} v_{i}^{-1} u_{2 n-2}^{-1}$ if $j=2 n-2$, and so we recover equations (4.4) and (4.5).

From the relator $\sigma_{1}^{j-1} \sigma_{3} \sigma_{2} \sigma_{3} \sigma_{2}^{-1} \sigma_{3}^{-1} \sigma_{2}^{-1} \sigma_{1}^{-(j-1)}$, we obtain the relators

$$
\begin{aligned}
& v_{1} u_{j+1} v_{1} u_{j+2}^{-1} v_{1}^{-1} u_{j}^{-1} \quad \text { if } j=1, \ldots, 2 n-4, \\
& v_{1} u_{2 n-2} v_{1} w u_{1}^{-1} w^{-1} v_{1}^{-1} u_{2 n-3}^{-1} \quad \text { if } j=2 n-3, \text { and } \\
& v_{1} w u_{1} v_{1} u_{2}^{-1} w^{-1} v_{1}^{-1} u_{2 n-2}^{-1} \quad \text { if } j=2 n-2,
\end{aligned}
$$

which yield respectively equations (4.6), (4.7) and (4.8).

Finally,

$$
\begin{aligned}
\sigma_{1} \sigma_{2} \cdots \sigma_{n-2} \sigma_{n-1}^{2} \sigma_{n-2} \cdots \sigma_{2} \sigma_{1}= & \sigma_{1} \sigma_{2} \sigma_{1}^{-2} \cdot \sigma_{1}^{2} \sigma_{3} \sigma_{1}^{-3} \\
\cdots \sigma_{1}^{n-3} \sigma_{n-2} \sigma_{1}^{-(n-2)} & \cdot \sigma_{1}^{n-2} \sigma_{n-1} \sigma_{1}^{-(n-1)} \cdot \sigma_{1}^{n-1} \sigma_{n-1} \sigma_{1}^{-n} \\
& \cdot \sigma_{1}^{n} \sigma_{n-2} \sigma_{1}^{-(n+1)} \cdot \sigma_{1}^{2 n-4} \sigma_{2} \sigma_{1}^{-(2 n-3)} \cdot \sigma_{1}^{2 n-2},
\end{aligned}
$$

and conjugating by $\sigma_{1}^{j-1}$, we obtain relators

$$
\begin{cases}u_{2}\left(v_{1} \cdots v_{n-4} v_{n-3}^{2} v_{n-4} \cdots v_{1}\right) u_{2 n-3} w & \text { if } j=1, \\ u_{3}\left(v_{1} \cdots v_{n-4} v_{n-3}^{2} v_{n-4} \cdots v_{1}\right) u_{2 n-2} w & \text { if } j=2, \\ u_{j+1}\left(v_{1} \cdots v_{n-4} v_{n-3}^{2} v_{n-4} \cdots v_{1}\right) w u_{j-2} & \text { if } j=3, \ldots, 2 n-3, \\ w u_{1}\left(v_{1} \cdots v_{n-4} v_{n-3}^{2} v_{n-4} \cdots v_{1}\right) u_{2 n-4} & \text { if } j=2 n-2 .\end{cases}
$$

This yields the remaining equations (4.12), (4.13), (4.14) and (4.15).

We now simplify somewhat the presentation of $\Gamma_{2}\left(B_{n}\left(\mathbb{S}^{2}\right)\right)$ given by Proposition 4.1. From equations (4.4) and (4.9), for $i=1,2$ we obtain the following 
equations:

(4.4.1)

$$
\begin{aligned}
& u_{1} v_{j}=v_{j} u_{2} \quad \text { for all } \quad j \geq 2, \\
& u_{2} v_{j}=v_{j} u_{1}^{-1} u_{2} \quad \text { for all } \quad j \geq 2 .
\end{aligned}
$$

This allows us to eliminate equation (4.5) as follows. For all $j \geq 2$, we have

$$
\begin{aligned}
v_{j} w u_{1} w^{-1} v_{j}^{-1} & =w v_{j} u_{1}^{-1} v_{j}^{-1} w^{-1} \quad \text { by equation (4.3) } \\
& \left.=w v_{j} u_{2} v_{j}^{-1} u_{2}^{-1} w^{-1} \quad \text { by equation (4.4 }\right) \\
& =w u_{1} u_{2}^{-1} w^{-1} \quad \text { by equation (4.4.) } \\
& =u_{2 n-2} \quad \text { by equation (4.11), }
\end{aligned}
$$

and this is equivalent to equation (4.5), which we thus delete from the list of relations.

Suppose that for some $2 \leq i \leq 2 n-4$, we have equations $(4.4-1)$ and (4.4). We now show that they imply $4.4 ;+1)$. For all $j \geq 2$, we have

$$
\begin{aligned}
u_{i+1} v_{j} u_{i+2}^{-1} v_{j}^{-1} & =u_{i-1}^{-1} u_{i} v_{j} u_{i+1}^{-1} u_{i} v_{j}^{-1} \quad \text { by equation (4.9) } \\
& =u_{i-1}^{-1} v_{j} u_{i} v_{j}^{-1} \quad \text { by equation (4.4) } \\
& =1 \quad \text { by equation (4.4 }-1 \text { ) }
\end{aligned}
$$

which yields equation $(4.4+1)$. So we may successively delete equations $(4.4 n-3)$, $\left(4.4 b_{n}-4\right), \ldots, 4.4$ ) from the list of relations.

We now show that we may delete all but one of the surface relations (4.12)(4.15). First suppose that we have equation (4.12). Now

$$
\begin{aligned}
u_{2 n-2} w u_{3} & =u_{2 n-3} w u_{1} u_{3} \quad \text { by equation (4.10) } \\
& \left.=u_{2 n-3} w u_{2} \quad \text { by equation (4.9 }\right) .
\end{aligned}
$$

This implies equation (4.13), which we delete from the list of relations.

Now suppose that we have equation $[4.14+1)$ for some $5 \leq i \leq 2 n-2$. Let us write $A=v_{1} \cdots v_{n-4} v_{n-3}^{2} v_{n-4} \cdots v_{1}$. Then $w u_{i-2} u_{i+1}=A^{-1}$. So

$$
\begin{aligned}
w u_{i-3} u_{i} & =w u_{i-2} u_{i+1} \quad \text { by equation }[4.911) \\
& =A^{-1} \quad \text { by above. }
\end{aligned}
$$

This yields equation (4.14;), and so we may delete successively equations $(4.14)$, $\ldots, 4.14 n-3)$.

Now suppose that we have (4.15), so $A u_{2 n-4} w u_{1}=1$. Then

$$
\begin{aligned}
A w u_{2 n-5} u_{2 n-2} & =A w u_{2 n-4} w u_{1} w^{-1} \quad \text { by equations (4.9) and (4.10) } \\
& =w\left(A u_{2 n-4} w u_{1}\right) w^{-1} \quad \text { by equation (4.3) } \\
& =1 \quad \text { by above. }
\end{aligned}
$$

This implies equation $\left(4.14 b_{n-2}\right)$, which we delete from the list of relations.

Finally, suppose that we have equation (4.12). Then

$$
\begin{aligned}
A u_{2 n-4} w u_{1} & \left.=A u_{2 n-3} u_{2 n-2}^{-1} w u_{1} \quad \text { by equation (4.9 }{ }_{n-4}\right) \\
& =A u_{2 n-3} w u_{2} \quad \text { by equation (4.11) } \\
& =1 \quad \text { by above. }
\end{aligned}
$$

This yields equation (4.15), which we delete from the list. It thus follows that we may delete all but one of the surface relations; let us keep equation (4.12). 
Summing up, we may thus delete relations (4.5), (4.4) for $i=3, \ldots, 2 n-3$ and (4.13) - (4.15) from the presentation of $\Gamma_{2}\left(B_{n}\left(\mathbb{S}^{2}\right)\right)$ given by Proposition 4.1.

4.2. The derived subgroup of $B_{4}\left(\mathbb{S}^{2}\right)$. The aim of this section is to use Proposition 4.1 to derive the presentation of $\Gamma_{2}\left(B_{4}\left(\mathbb{S}^{2}\right)\right)$ given in Theorem 1.3(3), from which we were able to see that $\Gamma_{2}\left(B_{4}\left(\mathbb{S}^{2}\right)\right) \cong \mathbb{F}_{2} \rtimes \mathcal{Q}_{8}$.

We first remark that in this case, the relations (4.1), (4.2), (4.4) and (4.5) do not exist. Further, from relations (4.9), we may obtain the following:

$$
u_{2}=u_{3} u_{4}^{-1} \quad u_{1}=u_{3} u_{4}^{-1} u_{3}^{-1} \quad u_{5}=u_{3}^{-1} u_{4} \quad u_{6}=u_{4}^{-1} u_{3}^{-1} u_{4},
$$

which we take to be definitions of $u_{1}, u_{2}, u_{5}$ and $u_{6}$, so we delete equation (4.9) from the list of relations. From equation (4.12), we see that

$$
w=u_{4}^{-1} u_{3} v_{1}^{-2} u_{4} u_{3}^{-1} .
$$

We conclude that $\Gamma_{2}\left(B_{4}\left(\mathbb{S}^{2}\right)\right)$ is generated by $u_{3}, u_{4}$ and $v_{1}$.

Let us return momentarily to the situation of the previous section. Before deleting all but one of the surface relations, we shall derive some other useful relations.

Consider the surface relations (4.12)-(4.15). From relations (4.12) and (4.14) (resp. (4.15) and (4.144)), it follows that $u_{5}$ (resp. $\left.u_{4}\right)$ commutes with $v_{1}^{2}$. But these two equations are equivalent to the relations

$$
\begin{aligned}
& u_{3} \rightleftharpoons v_{1}^{2} \quad \text { and } \\
& u_{4} \rightleftharpoons v_{1}^{2} .
\end{aligned}
$$

Further, equations (4.15) and (4.17) imply equation (4.144), and equations (4.12), (4.16) and (4.17) imply equation (4.14), so we replace equations $(4.144)$ and (4.145) by equations (4.16) and (4.17).

As in Section 4.1 we can then delete equations (4.14) and (4.15) from the list of relations, which becomes: (4.3), (4.6), (4.7), (4.8), (4.10) and (4.11). We now analyse these relations in further detail.

From equation (4.3) and the definition of $w$, we see that $v_{1} \rightleftharpoons u_{4}^{-1} u_{3} u_{4} u_{3}^{-1}$. Up to conjugacy, equation (4.6) may be written as follows:

$$
\begin{aligned}
1 & =u_{3} v_{1}^{-1} u_{3}^{-1} u_{3} u_{4} u_{3}^{-1} v_{1}^{-1} u_{3} u_{4}^{-1} u_{3}^{-1} v_{1}=u_{3} v_{1}^{-1} u_{3}^{-1} u_{4} u_{4}^{-1} u_{3} u_{4} u_{3}^{-1} v_{1}^{-1} u_{3} u_{4}^{-1} u_{3}^{-1} v_{1} \\
& =u_{3} v_{1}^{-1} u_{3}^{-1} u_{4} v_{1}^{-1} u_{4}^{-1} v_{1},
\end{aligned}
$$

and hence we may replace equation (4.6 $)$ by

$$
u_{3} v_{1} u_{3}^{-1}=u_{4} v_{1}^{-1} u_{4}^{-1} v_{1} \text {. }
$$

Up to conjugacy, equation (4.6b) may be written:

$$
u_{3}^{-1} v_{1} u_{3}=u_{4}^{-1} v_{1} u_{4} v_{1}^{-1} \text {. }
$$

By equations (4.18) and (4.16), the left-hand side of equation (4.63) may be written:

$$
u_{3} v_{1} u_{3}^{-1} u_{4} v_{1}^{-1} u_{4}^{-1} v_{1}^{-1}=u_{3} v_{1}^{2} u_{3}^{-1} v_{1}^{-2}=1,
$$

so relation $\left(4.6 \mathrm{~B}_{3}\right)$ is automatically satisfied, and we thus delete it from the list.

Using the fact that $v_{1} \rightleftharpoons u_{4}^{-1} u_{3} u_{4} u_{3}^{-1}$, equation (4.64) may be written:

$$
\begin{aligned}
1 & =u_{4} v_{1} u_{4}^{-1} u_{3}^{-1} u_{4} v_{1}^{-1} u_{4}^{-1} u_{3} v_{1}^{-1}=u_{4} v_{1} u_{3}^{-1} u_{3} u_{4}^{-1} u_{3}^{-1} u_{4} v_{1}^{-1} u_{4}^{-1} u_{3} v_{1}^{-1} \\
& =u_{4} v_{1} u_{3}^{-1} v_{1}^{-1} u_{3} u_{4}^{-1} v_{1}^{-1},
\end{aligned}
$$

and from this we obtain equation (4.19), using the fact that $v_{1}^{2}$ commutes with $u_{4}$. So we delete equation (4.64) from the list. 
We now consider equation (4.3). Using equations (4.18) and (4.19), we obtain

$$
\begin{aligned}
1 & =u_{4}^{-1} u_{3} u_{4} u_{3}^{-1} v_{1} u_{3} u_{4}^{-1} u_{3}^{-1} u_{4} v_{1}^{-1}=u_{4}^{-1} u_{3} v_{1} u_{4} v_{1}^{-1} u_{4}^{-1} u_{3}^{-1} u_{4} v_{1}^{-1} \\
& =u_{4}^{-1} u_{3} v_{1} u_{4} v_{1}^{-1} u_{4}^{-1} v_{1} u_{3}^{-1} v_{1}^{-1} u_{4}=u_{4}^{-1} u_{3} v_{1} u_{3} v_{1} u_{3}^{-2} v_{1}^{-1} u_{4},
\end{aligned}
$$

which up to conjugacy, and using the fact that $v_{1}^{2}$ commutes with $u_{3}$, yields

$$
u_{3}^{-2} v_{1}^{-1} u_{3} v_{1}^{-1} u_{3} v_{1}^{-1} \cdot v_{1}^{4}=1 .
$$

We replace equation (4.3) by this relation.

From equations (4.16) and (4.17), the left-hand side of equations (4.10) and (4.11) collapses, and so we delete these equations from the list.

After immediate cancellations, equation (4.8) becomes

$$
\begin{aligned}
1 & =u_{4}^{-1} u_{3}^{-1} u_{4} v_{1} u_{4}^{-1} u_{3} v_{1}^{-1} u_{4} v_{1}^{-1}=u_{4}^{-1} u_{3}^{-1} u_{4} v_{1} u_{4}^{-1} v_{1}^{-1} v_{1} u_{3} v_{1}^{-1} u_{4} v_{1} u_{4}^{-1} u_{4} v_{1}^{-2} \\
& =u_{4}^{-1} u_{3}^{-1} u_{3} v_{1} u_{3}^{-1} v_{1} u_{3} u_{3} v_{1}^{-1} u_{3}^{-1} u_{4} v_{1}^{-2}
\end{aligned}
$$

which up to conjugacy and inversion yields equation (4.20). So we delete equation (4.8) from the list.

After immediate cancellations, the left-hand side of equation (4.7) becomes

$$
\begin{aligned}
u_{3}^{-1} u_{4} v_{1} u_{4}^{-1} u_{3} u_{4}^{-1} u_{3}^{-1} u_{4} v_{1}^{-1} u_{4}^{-1} & =u_{3} u_{4} v_{1}^{-1}=u_{3}^{-1} u_{4} v_{1} u_{4}^{-1} v_{1}^{-1} u_{3} v_{1}^{-1} \\
& =u_{3}^{-1} u_{3} v_{1} u_{3}^{-1} u_{3} v_{1}^{-1}=1,
\end{aligned}
$$

using the fact that $v_{1} \rightleftharpoons u_{4}^{-1} u_{3} u_{4} u_{3}^{-1}$ and applying equations (4.18) and (4.17). So we delete equation (4.7) from the list.

We are thus left with relations (4.16), (4.17), (4.18), (4.19) and (4.20). We now multiply together equations (4.18) and (4.19). The product of the left-hand sides, by equation (4.20), is given by

$$
u_{3} v_{1} u_{3}^{-2} v_{1} u_{3}=v_{1}
$$

while by equations (4.16), (4.17), (4.18), (4.19) and (4.20), the product of the right-hand sides is given by

$$
\begin{aligned}
u_{4} v_{1}^{-1} u_{4}^{-1} v_{1} u_{4}^{-1} v_{1} u_{4} v_{1}^{-1} & =u_{4} v_{1}^{-1} u_{4}^{-1} v_{1} u_{4}^{-1} v_{1}^{-1} u_{4} v_{1}=v_{1}^{-1} v_{1} u_{4} v_{1}^{-1} u_{4}^{-1} u_{3}^{-1} v_{1}^{-1} u_{3} v_{1} \\
& =v_{1}^{-1} v_{1}^{-1} u_{4} v_{1} u_{4}^{-1} u_{3}^{-1} v_{1}^{-1} u_{3} v_{1}=v_{1}^{-1} u_{3} v_{1}^{-1} u_{3}^{-2} v_{1}^{-1} u_{3} v_{1} \\
& =v_{1}^{-3} .
\end{aligned}
$$

From these two equations, we conclude that

$$
v_{1}^{4}=1
$$

and so equation (4.20) becomes

$$
u_{3}^{-2} v_{1}^{-1} u_{3} v_{1}^{-1} u_{3} v_{1}^{-1}=1
$$

The list of relations now becomes (4.16), (4.17), (4.21), (4.22), (4.18) and (4.19). We may rewrite the corresponding presentation as follows:

Proposition 4.3. The following constitutes a presentation of the group $\Gamma_{2}\left(B_{4}\left(\mathbb{S}^{2}\right)\right)$ : generators: $g_{1}, g_{2}, g_{3}$, where in terms of the usual generators of $B_{4}\left(\mathbb{S}^{2}\right), g_{1}=$ $u_{3}=\sigma_{1}^{2} \sigma_{2} \sigma_{1}^{-3}, g_{2}=u_{4}=\sigma_{1}^{3} \sigma_{2} \sigma_{1}^{-4}$ and $g_{3}=v_{1}=\sigma_{3} \sigma_{1}^{-1}$. 


\section{relations:}

$$
\begin{gathered}
g_{3}^{4}=1, \\
g_{3}^{2} \rightleftharpoons g_{1}, \\
g_{3}^{2} \rightleftharpoons g_{2}, \\
g_{3} \rightleftharpoons g_{2} g_{1}, \\
g_{2}^{-1} g_{1}^{-1} g_{3}^{-1} g_{1} g_{2} g_{3}^{-1}=1, \\
g_{1}^{-2} g_{3}^{-1} g_{1} g_{3}^{-1} g_{1} g_{3}^{-1}=1 .
\end{gathered}
$$

Proof. Rewriting $u_{3}, u_{4}$ and $v_{1}$ in terms of the $g_{i}$, we obtain directly the first three and the last of the given relations. As for the fourth and fifth relations, we obtain respectively

$$
\begin{aligned}
g_{3} g_{2} g_{1} g_{3}^{-1} g_{1}^{-1} g_{2}^{-1} & =v_{1} u_{4} u_{3}^{-1} v_{1}^{-1} u_{3}^{-1} u_{4}^{-1}=u_{4}\left(u_{4}^{-1} v_{1} u_{4} v_{1}^{-1} v_{1} u_{3}^{-1} v_{1}^{-1} u_{3}^{-1}\right) u_{4}^{-1} \\
& =u_{4} u_{3}\left(u_{3}^{-2} v_{1}^{-1} u_{3} v_{1}^{-1} u_{3} v_{1}^{-1} v_{1}^{4}\right) u_{3}^{-1} u_{4}^{-1}=1
\end{aligned}
$$

by equations (4.19), (4.21) and (4.22), and

$$
\begin{aligned}
g_{2}^{-1} g_{1}^{-1} g_{3}^{-1} g_{1} g_{2} g_{3}^{-1} & =u_{4}^{-1} u_{3}^{-1} v_{1}^{-1} u_{3} u_{4} v_{1}^{-1} \\
& =v_{1} u_{4}^{-1}\left(u_{4} v_{1}^{-1} u_{4}^{-1} v_{1} v_{1}^{-1} u_{3}^{-1} v_{1}^{-1} u_{3}\right) u_{4} v_{1}^{-1} \\
& =v_{1} u_{4}^{-1} u_{3}\left(v_{1} u_{3}^{-1} v_{1}^{-1} u_{3}^{-1} v_{1}^{-1} u_{3}^{2}\right) u_{3}^{-1} u_{4} v_{1}^{-1}=1
\end{aligned}
$$

by equations (4.18), (4.21) and (4.22). Thus the presentation we derived with generators $u_{3}, u_{4}$ and $v_{1}$ implies the system given by Proposition 4.3. Conversely, given this system, we have

$$
u_{3} v_{1} u_{3}^{-1}=u_{3}^{-1} v_{1}^{-1} u_{3} v_{1}^{-1}=u_{4} v_{1}^{-1} u_{4}^{-1} v_{1},
$$

which is equation (4.18), and

$$
u_{3}^{-1} v_{1} u_{3}=u_{3}^{-1} v_{1}^{-1} u_{3} v_{1}^{2}=u_{3} v_{1} u_{3}^{-1} v_{1} v_{1}^{2}=u_{4}^{-1} v_{1} u_{4} v_{1}^{3}=u_{4}^{-1} v_{1} u_{4} v_{1}^{-1},
$$

which is equation (4.19). Hence the system given by Proposition 4.3 is equivalent to our presentation with generators $u_{3}, u_{4}$ and $v_{1}$, and so in particular is a presentation of $\Gamma_{2}\left(B_{4}\left(\mathbb{S}^{2}\right)\right)$.

\section{ACKNOWLEDGEMENTS}

This work took place during the visit of the second author to the Departmento de Matemática do IME-Universidade de São Paulo during the periods $5^{\text {th }}-31^{\text {st }}$ August $2003,9^{\text {th }}$ July $-4^{\text {th }}$ August 2004, and $23^{\text {rd }}$ June $-23^{\text {rd }}$ July 2005, and of the visit of the first author to the Laboratoire de Mathématiques Emile Picard during the period $30^{\text {th }}$ September $-1^{\text {st }}$ November 2004. The first and fourth of these visits were supported by the international Cooperation Capes/Cofecub project number 364/01. The second visit was supported by the 'Accord franco-brésilien en mathématiques', and the third visit by FAPESP, Projeto Temático Topologia Algébrica, Geométrica e Differencial-2000/05385-8. 


\section{REFERENCES}

1. E. Artin, Theorie der Zöpfe, Abh. Math. Sem. Univ. Hamburg 4 (1925), 47-72.

2. _ Theory of braids, Ann. Math. 48 (1947), 101-126. MR0019087 (8:367a)

3. _ Braids and permutations, Ann. Math. 48 (1947), 643-649. MR0020989 (9:6c)

4. P. Bellingeri, S. Gervais and J. Guaschi, Lower central series of Artin-Tits and surface braid groups, J. Algebra 319 (2008), 1409-1427. MR2383053

5. J. S. Birman, On braid groups, Comm. Pure and Appl. Math. 22 (1969), 41-72. MR0234447 $(38: 2764)$

6. , Braids, links and mapping class groups, Ann. Math. Stud. 82, Princeton University Press, 1974. MR0375281(51:11477)

7. - Mapping class groups of surfaces, in Braids (Santa Cruz, CA, 1986), 13-43, Contemp. Math. 78, Amer. Math. Soc., Providence, RI, 1988. MR975076 (90g:57013)

8. K. S. Brown, Cohomology of groups, Graduate Texts in Mathematics, 87, Springer-Verlag, New York, 1982. MR672956 (83k:20002)

9. G. Burde and H. Zieschang, Knots, Second edition, de Gruyter Studies in Mathematics, 5. Walter de Gruyter \& Co., Berlin, 2003. MR1959408 (2003m:57005)

10. F. R. Cohen and S. Gitler, On loop spaces of configuration spaces, Trans. Amer. Math. Soc. 354 (2002), 1705-1748. MR1881013 (2002m:55020)

11. E. Fadell, Homotopy groups of configuration spaces and the string problem of Dirac, Duke Math. Journal 29 (1962), 231-242. MR0141127|(25:4538)

12. E. Fadell and S. Y. Husseini, Geometry and topology of configuration spaces, Springer Monographs in Mathematics. Springer-Verlag, Berlin, 2001. MR1802644 (2002k:55038)

13. E. Fadell and L. Neuwirth, Configuration spaces, Math. Scandinavica 10 (1962), 111-118. MR 0141126 (25:4537)

14. E. Fadell and J. Van Buskirk, The braid groups of $\mathbb{E}^{2}$ and $\mathbb{S}^{2}$, Duke Math. Journal 29 (1962), 243-257. MR0141128 (25:4539)

15. M. Falk and R. Randell, The lower central series of a fiber-type arrangement, Invent. Math. 82 (1985), 77-88. MR808110 (87c:32015b)

16. - The lower central series of generalized pure braid groups, in Geometry and topology (Athens, Ga., 1985), 103-108, Lecture Notes in Pure and Appl. Math. 105, Dekker, New York, 1987. MR873287 (88c:20048)

17. _ Pure braid groups and products of free groups, in Braids (Santa Cruz, CA, 1986), 217228, Contemp. Math. 78, Amer. Math. Soc., Providence, RI, 1988. MR975081 (90d:20070)

18. R. H. Fox and L. Neuwirth, The braid groups, Math. Scandinavica 10 (1962), 119-126. MR0150755 (27:742)

19. R. Gillette and J. Van Buskirk, The word problem and consequences for the braid groups and mapping class groups of the 2-sphere, Trans. Amer. Math. Soc. 131 (1968), 277-296. MR.0231894(38:221)

20. D. L. Gonçalves and J. Guaschi, On the structure of surface pure braid groups, J. Pure Appl. Algebra 182 (2003), 33-64 (due to a printer's error, this article was republished in its entirety in 186 (2004), 187-218). MR.1977999 (2004i:20068)

21. 137 (2004), 307-320. MR2092062 (2005i:20060)

22. 757-780. MR2100679 (2005j:20040)

23. - The braid group $B_{n, m}\left(\mathbb{S}^{2}\right)$ and the generalised Fadell-Neuwirth short exact sequence, J. Knot Theory and its Ramifications 14 (2005), 375-403. MR2149513 (2006b:20052)

24. - The quaternion group as a subgroup of the sphere braid groups, Bull. London Math. Soc. 39 (2007), 232-234. MR2323453 (2008b:20041)

25. - The braid groups of the projective plane and the Fadell-Neuwirth short exact sequence, Geom. Dedicata 130 (2007), 93-107. MR2365780

26. The classification and the conjugacy classes of the finite subgroups of the sphere braid groups, Algebraic and Geometric Topology 8 (2008), 757-785.

27. sphere, to appear in J. Knot Theory and its Ramifications.

28. Classification of the virtually cyclic subgroups of the pure braid groups of the projective plane, preprint October 2007, arXiv:math.GT/0710.5940. 
29. _ Classification of the virtually cyclic subgroups of the sphere braid groups, work in progress.

30. Classification of the finite and virtually cyclic subgroups of the braid groups of the projective plane, work in progress.

31. J. González-Meneses and L. Paris, Vassiliev invariants for braids on surfaces, Trans. Amer. Math. Soc. 356 (2004), 219-243. MR2020030 (2004m:20073)

32. E. A. Gorin and V Ja. Lin, Algebraic equations with continuous coefficients and some problems of the algebraic theory of braids, Math. USSR Sbornik 7 (1969), 569-596.

33. M. Hall, The theory of groups, Macmillan, New York, 1959. MR0103215 (21:1996)

34. V. L. Hansen, Braids and Coverings: Selected topics, London Math. Society Student Text, 18, Cambridge University Press, 1989. MR1247697 (94g:57004)

35. P. Hilton, G. Mislin and L. Roitberg, Localization of nilpotent groups and spaces, NorthHolland Mathematics Studies, No. 15, Notas de Matemática, No. 55, North-Holland Publishing Co., Amsterdam, 1975. MR0478146(57:17635)

36. T. Kohno, Série de Poincaré-Koszul associée aux groupes de tresses pures, Invent. Math. 82 (1985), 57-75. MR808109 (87c:32015a)

37. W. Magnus, A. Karrass and D. Solitar, Combinatorial group theory, Second revised edition, Dover Publications, Inc., New York, 1976. MR0422434 (54:10423)

38. K. Murasugi, Seifert fibre spaces and braid groups, Proc. London Math. Soc. 44 (1982), 71-84. MR642793 (83f:57007)

39. L. Paris and D. Rolfsen, Geometric subgroups of surface braid groups, Ann. Inst. Fourier 49 (1999), 417-472. MR1697370 (2000f:20059)

40. D. Rolfsen, New developments in the theory of Artin's braid groups, Proceedings of the Pacific Institute for the Mathematical Sciences Workshop 'Invariants of Three-Manifolds' (Calgary, AB, 1999), Topology and its Applications 127 (2003), 77-90. MR.1953321 (2004f:20070)

41. G. P. Scott, Braid groups and the group of homeomorphisms of a surface, Proc. Camb. Phil. Soc. 68 (1970), 605-617. MR0268889(42:3786)

42. J. Stallings, Homology and central series of groups, J. Algebra 2 (1965), 170-181. MR0175956 $(31: 232)$

43. J. G. Thompson, Note on H(4), Comm. Algebra 22 (1994), 5683-5687. MR 1298742 (95h:20048)

44. J. Van Buskirk, Braid groups of compact 2-manifolds with elements of finite order, Trans. Amer. Math. Soc. 122 (1966), 81-97. MR0189013(32:6440)

45. O. Zariski, On the Poincaré group of rational plane curves, Amer. J. Math. 58 (1936), 607619. MR 1507185

46. 59 (1937), 335-358. MR 1507244

Departamento de Matemática, Instituto de Matemática e Estatística, Universidade de Saõ Paulo, Caixa Postal 66281, Ag. Cidade de São Paulo, CeP: 05314-970, São Paulo, SP, BRAZIL

E-mail address: dlgoncal@ime.usp.br

Laboratoire de Mathématiques Emile Picard, UMr CNRS 5580, UFR-Mig, Université Toulouse III, 31062 Toulouse Cedex 9, France

Current address: Laboratoire de Mathématiques Nicolas Oresme, UMR CNRS 6139, Université de Caen BP 5186, 14032 Caen Cedex, France

E-mail address: guaschi@math.unicaen.fr 